\title{
Analytical solution of the tokamak equilibrium. II. The free-boundary case
}

\author{
G. O. Ludwig ${ }^{\text {a) }}$ \\ Departamento de Física, Instituto Tecnológico de Aeronáutica, Comando da Aeronáutica 12228-900 São José \\ dos Campos, SP, Brazil
}

(Received 16 May 2017; accepted 25 July 2017; published online 10 August 2017)

\begin{abstract}
The free-boundary tokamak equilibrium problem is investigated by a surface current equivalence method applied on the plasma boundary. In addition, use is made of a spectral representation for the internal plasma flux surfaces as presented in Paper I [G. O. Ludwig, Phys. Plasma 24, 092502 (2017)]. The surface current distribution is determined by the Cauchy condition imposed by the external equilibrium coils on the plasma boundary. A self-consistent approximate analytic equilibrium is calculated for an up-down asymmetric configuration of the plasma contained by a simplified set of poloidal field coils representing an ITER-like tokamak. Published by AIP Publishing.

[http://dx.doi.org/10.1063/1.4997794]
\end{abstract}

\section{INTRODUCTION}

The design of tokamak devices depends on detailed magnetohydrodynamic equilibrium calculations of axisymmetric plasma configurations. In particular, the design depends on the determination of currents in external poloidal field coils that contain plasma of a specified cross-sectional shape. The calculations must include the corrections in the plasma shape necessary to make the equilibrium consistent with the field produced by a finite set of current-carrying coils.

Little pure analytical work has been done on the freeboundary problem. The solution of the problem is formulated in its general form by means of the virtual-casing principle and the containing magnetic field concept. ${ }^{2-4}$ Early approximate plasma equilibria in external multipole configurations were determined in order to analyze free-boundary configurations for a plasma ring. ${ }^{5}$ However, the toroidal current density distribution in this early model is not self-consistent because it is assumed uniform inside the plasma, but the vacuum self-field is calculated for a current loop centered on the magnetic axis. Free-boundary equilibria of sharp-boundary tokamak plasmas inside a circular conducting shell were solved in the high-beta approximation using conformal mapping and Fourier transformations. ${ }^{6}$ The sharp-boundary model reduces the plasma description to a constant pressure volume surrounded by skin currents. This restriction has been partly removed in further advances of the conformal mapping methods, ${ }^{7}$ but complications remain for arbitrary, noncircular cross-sections, and the method has not been applied for finite sets of equilibrium coils. Usually, the external equilibrium solution in an analytical work is described using orthogonal toroidal coordinates and large aspect ratio expansions. ${ }^{4,7}$

Due to the inherent nonlinearity, the free-boundary problem has been treated mostly using fully numerical codes. Early numerical efforts using iterative methods ${ }^{9,10}$ originated a multitude of equilibrium solvers presently used in the design of modern tokamaks. A detailed account of the numerical methods used in these codes is presented in Ref. 11.

\footnotetext{
${ }^{a}$ E-mail: gerson.ludwig@inpe.br
}

In the present article, the "direct" and "inverse" aspects of the free-boundary problem are reexamined taking advantage of both the equivalent surface current density description, closely related to the virtual-casing principle, and the analytic simplifications introduced by a spectral representation of the plasma flux surfaces. The spectral representation is detailed in Paper I, where it was used to determine the approximate internal equilibrium solutions of an ITER-like tokamak. In Sec. II of the present article, the ill-posed "direct" problem, related to the external equilibrium field solution is reviewed, and the currents in a system of simplified poloidal coils of the ITERlike tokamak are approximately calculated in order to contain the initially determined internal equilibrium. Due to the finite number of external coils, this containment is not fully adequate, and an adjustment of the plasma boundary conditions is necessary. This problem is dealt with in Sec. III, where the equivalent surface current density on the plasma boundary is determined in consistency with the containing field imposed by the external coils. In principle, the free-boundary equilibrium problem is solved at this stage because the adjusted surface current density fully determines the internal plasma current density distribution. This "inverse" problem for the plasma current density can be solved by the introduction of appropriate models for the internal solution, determining the self-consistent current distribution in an approximate form only. The adjustment of the internal equilibrium flux surfaces and the related current distribution in the ITER-like tokamak is carried out in Sec. IV. As a further application, the poloidal flux and the equivalent surface current density distributions on the plasma boundary, corresponding to the start of burn conditions of ITER, are determined in Sec. V. Section VI gives the conclusions.

\section{EXTERNAL EQUILIBRIUM FIELD ADJUSTMENT}

The "direct" fixed-boundary equilibrium problem involves the determination in the entire space of the equilibrium magnetic field $\vec{B}=\vec{B}_{\text {self }}+\vec{B}_{\text {ext }}$ formed by the plasma self-field $\vec{B}_{\text {self }}$ and the containing field $\vec{B}_{\text {ext }}$ produced by external sources, for a given plasma shape. The approximate analytic solution of the Grad-Shafranov equation, presented in Paper $\mathrm{I}^{1}{ }^{1}$ 
completely specifies the internal equilibrium magnetic field, that is, the self-field $\vec{B}_{\text {self }}$ associated with the plasma current, and the containing field $\vec{B}_{\text {ext }}$ at the plasma boundary. Now, in order to solve the fixed-boundary problem in all space, the equilibrium field must be extended beyond the plasma boundary. The extension of this field into a vacuum constitutes an ill-posed problem, requiring the solution of a Cauchy-type initial-value problem for an elliptic partial differential equation. Nevertheless, an approximate solution can be found using the integral formulation of the problem and minimizing the quadratic error between the exact containing field $\vec{B}_{\text {ext }}$, determined from the internal solution, and the field produced near the plasma boundary by either an external surface current distribution or currents in a finite set of external coils.

Using the definitions and notation introduced in Sec. IV of Paper I, the total poloidal flux function $\Phi=\Phi_{0}-\Phi_{P}$ between the symmetry axis and a given flux surface is given in terms of the toroidal current density $j_{T}$ by Ampère's law in flux coordinates $(\rho, \theta, \zeta)$

$$
\Delta^{2} \Phi=h_{\zeta}^{2} \nabla \cdot\left(h_{\zeta}^{-2} \nabla \Phi\right)=-2 \pi \mu_{0} h_{\zeta} j_{T}
$$

The flux linked by the magnetic axis is denoted by $\Phi_{0}$, and $\Phi_{P}$ is the poloidal flux between the magnetic axis and the flux surface, which is produced by the toroidal plasma current. This equation for $\Phi$ can be formally solved in terms of the Green's function for an impulsive toroidal ring current, defined by

$$
\left\{\begin{array}{l}
\Delta^{2} G\left(\vec{r} ; \vec{r}^{\prime}\right)=-2 \pi h_{\zeta} \delta^{2}\left(\vec{r}-\vec{r}^{\prime}\right) \\
\delta^{2}\left(\vec{r}-\vec{r}^{\prime}\right)=2 \pi h_{\zeta}^{\prime} \delta^{3}\left(\vec{r}-\vec{r}^{\prime}\right)
\end{array}\right.
$$

Here, the two-dimensional delta function is normalized in such a way that

$$
\iint_{S^{\prime} \rightarrow \infty} \delta^{2}\left(\vec{r}-\vec{r}^{\prime}\right) d^{2} r\left(\zeta^{\prime}\right)=1
$$

where $d^{2} r(\zeta)=\left(\sqrt{g} / h_{\zeta}\right) d \rho d \theta$ is the differential area element in the coordinate surface $\zeta=$ constant, and the quotation mark indicates the coordinates of the sources.

From the Biot-Savart law for the vector potential, the Green's function for the axisymmetric Ampère's equation may be written as

$$
G\left(\vec{r} ; \vec{r}^{\prime}\right)=\left\langle\frac{\pi h_{\zeta} h_{\zeta}^{\prime} \cos \left(\Delta \zeta^{\prime}\right)}{\left|\vec{r}-\vec{r}^{\prime}\right|}\right\rangle_{\Delta \zeta^{\prime}},
$$

where $\langle\ldots\rangle_{\zeta}=(2 \pi)^{-1} \int_{0}^{2 \pi}(\ldots) d \zeta$ denotes the toroidal angle average. It follows that the flux function is given by the surface integral

$$
\Phi(\vec{r})=\mu_{0} \iint_{S_{T}^{\prime}} j_{T}\left(\vec{r}^{\prime}\right) G\left(\vec{r} ; \vec{r}^{\prime}\right) d^{2} r\left(\zeta^{\prime}\right) .
$$

Consider a general solution $\psi$ of the homogeneous equation $\Delta^{2} \psi=0$ of volume $V$. Multiplying Ampère's law by $\psi / h_{\zeta}^{2}$ and the homogeneous equation by $\Phi / h_{\zeta}^{2}$, subtracting the results, integrating over the total volume $V(a)$ of the plasma, and applying Green's second integral identity with azimuthal symmetry, one obtains

$$
\iint_{S_{T}(a)} j_{T} \psi d^{2} r(\zeta)=\oint_{\ell_{P}(a)}\left(K_{T} \psi+\frac{\Phi(a)}{2 \pi \mu_{0} h_{\zeta}} \hat{n} \cdot \nabla \psi\right) d \ell(\theta),
$$

where $d \ell(\theta)=h_{\theta} d \theta$ is the differential arc length along the coordinate curve $\theta$, and $K_{T}=h_{\zeta} \vec{K} \cdot \nabla \zeta=-\hat{n} \cdot \nabla \Phi /\left(2 \pi \mu_{0} h_{\zeta}\right)$ defines the toroidal component of the equivalent surface current density $\vec{K}$. The second term on the right-hand side of this equation can be written as

$$
\begin{aligned}
& \Phi(a) \oint_{\ell_{P}(a)}\left(\frac{\hat{n} \cdot \nabla \psi}{2 \pi \mu_{0} h_{\zeta}}\right) d \ell(\theta) \\
& =\Phi(a) \oint_{\ell_{P}(a)}\left(\nabla \zeta \times \frac{\nabla \psi}{2 \pi \mu_{0}}\right) \cdot d \vec{\ell}(\theta) \\
& =-\Phi(a) \oint_{\ell_{P}(a)} \nabla \times\left(\frac{\psi}{2 \pi \mu_{0}} \nabla \zeta\right) \cdot d \vec{\ell}(\theta) \\
& =-\Phi(a) \oint_{\ell_{P}(a)} \frac{\vec{B}_{h}}{\mu_{0}} \cdot d \vec{\ell}(\theta)=0,
\end{aligned}
$$

where $\vec{B}_{h}=\nabla \times(\psi \nabla \zeta / 2 \pi)=-\nabla \zeta \times \nabla \psi / 2 \pi$ is the induction field corresponding to the axisymmetric flux function $\psi$. The line integral of $\vec{B}_{h} / \mu_{0}$ around the closed path $\ell_{P}(a)$ gives the linked current. This current is null for the homogeneous equation solution, whence the integral consistency condition

$$
\iint_{S_{T}(a)} j_{T} \psi d^{2} r(\zeta)=\oint_{\ell_{P}(a)} K_{T} \psi d \ell(\theta) .
$$

Taking $\psi=$ constant leads to the integral equivalence of $j_{T}$ and $K_{T}$ in terms of the total toroidal plasma current $I_{T}(a)$

$$
\iint_{S_{T}(a)} j_{T} d^{2} r(\zeta)=\oint_{\ell_{P}(a)} K_{T} d \ell(\theta)=I_{T}(a) .
$$

The integral consistency condition (8) corresponds to a contour integral taken exactly on a flux surface coinciding with the plasma boundary. With proper replacements $(a \rightarrow \rho)$, this condition is valid for any internal magnetic surface designated by the radial coordinate $\rho$. Furthermore, since Green's function may be expressed in terms of solutions of the homogeneous Ampère equation, the consistency condition allows writing the self-flux in the outside region (vacuum region) in terms of the equivalent surface current density $K_{T}(\theta)$ on the plasma boundary

$$
\Phi_{\text {self }}(\vec{r})=\mu_{0} \oint_{\ell_{P}(a)} K_{T}(\theta) G(\vec{r} ; \theta) d \ell(\theta) .
$$

The total poloidal flux in the outside region is given by $\Phi_{\text {self }}$ added to the poloidal flux $\Phi_{\text {ext }}$ produced by the external sources

$$
\Phi(\vec{r})=\mu_{0} \oint_{\ell_{P}(a)} K_{T}(\theta) G(\vec{r} ; \theta) d \ell(\theta)+\Phi_{\mathrm{ext}}(\vec{r})
$$

(vacuum region). 
At the plasma boundary $\rho=a$, the constant flux condition $\Phi(\theta)=\Phi(a)$ provides a link between the internal and external sources (Dirichlet condition)

$$
\begin{aligned}
& \Phi(a)=\mu_{0} \oint_{\ell_{P}(a)} K_{T}\left(\theta^{\prime}\right) G\left(\theta ; \theta^{\prime}\right) d \ell\left(\theta^{\prime}\right)+\Phi_{\text {ext }}(\theta) \\
& \text { (plasma boundary). }
\end{aligned}
$$

Just outside the plasma boundary, the total poloidal flux produced by the external sources can be written as

$$
\Phi_{\mathrm{ext}}^{(+)}(\theta)=\Phi(a)-\mu_{0} \oint_{\ell_{P}^{(+)}} K_{T}\left(\theta^{\prime}\right) G\left(\theta ; \theta^{\prime}\right) d \ell\left(\theta^{\prime}\right),
$$

where $\ell_{P}^{(+)}$designates a poloidal contour in the vacuum region near the plasma boundary.

In an axisymmetric system, the component of the magnetic field tangential to a flux surface is calculated in terms of the total poloidal flux $\Phi$ by

$$
B_{\tau}=h_{\zeta}(\hat{n} \times \vec{B}) \cdot \nabla \zeta=h_{\zeta}(\vec{B} \times \nabla \zeta) \cdot \hat{n}=-\frac{\hat{n} \cdot \nabla \Phi}{2 \pi h_{\zeta}},
$$

where $\hat{n}$ is the unit vector normal to the flux surface. Accordingly, just outside the plasma boundary, the tangential component of the magnetic field due to external sources is

$$
\begin{aligned}
\hat{n} \times \vec{B}_{\mathrm{ext}}^{(+)}(\theta) & =h_{\zeta}(\theta) B_{\tau, \mathrm{ext}}^{(+)}(\theta) \nabla \zeta \\
& =\left(\frac{\mu_{0}}{2 \pi} \oint_{\ell_{P}^{(+)}} K_{T}\left(\theta^{\prime}\right) \hat{n} \cdot \nabla G\left(\theta ; \theta^{\prime}\right) d \ell\left(\theta^{\prime}\right)\right) \nabla \zeta .
\end{aligned}
$$

The normal component of $\vec{B}$ is continuous across a surface layer of current $\vec{K}=h_{\zeta} K_{T} \nabla \zeta$, but the tangential component experiences a discontinuity defined by

$$
\hat{n} \times\left(\vec{B}_{(+)}-\vec{B}_{(-)}\right)=\mu_{0} \vec{K} .
$$

Therefore, the tangential component of the magnetic field due to external sources is given just inside the plasma boundary $\ell_{P}(a)$ by

$$
\begin{aligned}
B_{\tau, \text { ext }}^{(-)}(\theta)= & \frac{\mu_{0}}{2 \pi h_{\zeta}(\theta)} \oint_{\ell_{P}^{(+)}} K_{T}\left(\theta^{\prime}\right) \hat{n} \cdot \nabla G\left(\theta ; \theta^{\prime}\right) d \ell\left(\theta^{\prime}\right) \\
& -\mu_{0} K_{T}(\theta) .
\end{aligned}
$$

Integration over the boundary yields

$$
\begin{aligned}
\oint_{\ell_{P}(a)} B_{\tau, \mathrm{ext}}^{(-)}(\theta) d \ell(\theta)= & \mu_{0} \oint_{\ell_{P}^{++}} K_{T}\left(\theta^{\prime}\right) \\
& \times\left(\oint_{\ell_{P}(a)} \frac{\hat{n} \cdot \nabla G\left(\theta ; \theta^{\prime}\right)}{2 \pi h_{\zeta}(\theta)} d \ell(\theta)\right) d \ell\left(\theta^{\prime}\right) \\
& -\mu_{0} \oint_{\ell_{P}(a)} K_{T}(\theta) d \ell(\theta)
\end{aligned}
$$

where

$$
\oint_{\ell_{P}(a)} \frac{\hat{n} \cdot \nabla G\left(\theta ; \theta^{\prime}\right)}{2 \pi h_{\zeta}(\theta)} d \ell(\theta)= \begin{cases}1 & \text { inside } \\ 1 / 2 & \text { on the boundary } \\ 0 & \text { outside }\end{cases}
$$

in accordance with Gauss' theorem and Ampère's law. Hence, for integration exactly on the plasma boundary, the containing field can be written as (Neumann condition)

$$
\begin{aligned}
B_{\tau, \mathrm{ext}}(\theta)= & \frac{\mu_{0}}{2 \pi h_{\zeta}(\theta)} \oint_{\ell_{P}(a)} K_{T}\left(\theta^{\prime}\right) \hat{n} \cdot \nabla G\left(\theta ; \theta^{\prime}\right) d \ell\left(\theta^{\prime}\right) \\
& -\frac{\mu_{0} K_{T}(\theta)}{2} .
\end{aligned}
$$

Now, in order to extend the containing field into the vacuum region, consider the poloidal flux produced by an external surface current density $K_{T, \text { ext }}$ :

$$
\Phi_{\text {ext }}(\vec{r})=\mu_{0} \oint_{\ell} K_{T, \text { ext }}\left(\vec{r}^{\prime}\right) G\left(\vec{r} ; \vec{r}^{\prime}\right) d \ell\left(\vec{r}^{\prime}\right),
$$

where $\ell$ designates the external source support, which coincides with a flux surface in the vacuum region. This is an application of the virtual-casing principle. ${ }^{2}$ To attain equilibrium, the magnetic field produced by the current $K_{T \text {,ext }}$ flowing on $\ell$ must be such that its tangential component on $\ell_{P}(a)$ coincides with the containing field $B_{\tau, \text { ext }}(\theta)$ related to the current $K_{T}(\theta)$. Hence, the desired current $K_{T, \text { ext }}$ is determined by a Fredholm integral equation of the first kind

$$
\begin{aligned}
& \oint_{\ell^{\prime}} K_{T, \mathrm{ext}}\left(\vec{r}^{\prime}\right) \hat{n} \cdot \nabla G\left(\theta ; \vec{r}^{\prime}\right) d \ell\left(\vec{r}^{\prime}\right) \\
& \quad=\pi h_{\zeta}(\theta) K_{T}(\theta)-\oint_{\ell_{P}(a)} K_{T}\left(\theta^{\prime}\right) \hat{n} \cdot \nabla G\left(\theta ; \theta^{\prime}\right) d \ell\left(\theta^{\prime}\right),
\end{aligned}
$$

which constitutes an ill-posed problem, since the addition of a rapidly oscillating term to $K_{T, \text { ext }}$ will not change the value of the integral. This equation can be numerically solved for $K_{T, \text { ext }}$ by means of regularization techniques that eliminate the oscillating terms in the solution. Conversely, the above equation constitutes a usually well-conditioned Fredholm equation of second kind for $K_{T}(\theta)$.

The external current carrying surface is not required to be flux-conserving (superconducting) as in most firstgeneration short-pulsed tokamaks. An approximate solution for $K_{T, \text { ext }}$ can be obtained limiting the external sources to a finite set of coils. For a circular coil with current density

$$
j_{k}=I_{k} \delta^{2}\left(\vec{r}-\vec{r}_{k}\right), \quad \iint_{S_{k}} \delta^{2}\left(\vec{r}-\vec{r}_{k}\right) d S_{k}=1,
$$

the corresponding poloidal flux is

$$
\Phi_{k}(\vec{r})=\mu_{0} \iint_{S_{k}} j_{k}\left(\vec{r}^{\prime}\right) G\left(\vec{r} ; \vec{r}^{\prime}\right) d S_{k}^{\prime}=\mu_{0} I_{k} G\left(\vec{r} ; \vec{r}_{k}\right) .
$$

Each coil produces a magnetic field component tangential to $\ell_{P}(a)$ given by 


$$
B_{\tau, k}(\theta)=-\frac{\hat{n} \cdot \nabla \Phi_{k}}{2 \pi h_{\zeta}(\theta)}=-\frac{\mu_{0} I_{k}}{2 \pi h_{\zeta}(\theta)} \hat{n} \cdot \nabla G\left(\theta ; \vec{r}_{k}\right),
$$

so that the coils currents $I_{k}$ at fixed positions $\vec{r}_{k}$ can be determined by a least-squares technique in order to satisfy the approximate external equilibrium equation

$$
\begin{aligned}
\sum_{k} I_{k} \hat{n} \cdot \nabla G\left(\theta ; \vec{r}_{k}\right) \cong & \pi h_{\zeta}(\theta) K_{T}(\theta) \\
& -\oint_{\ell_{P}(a)} K_{T}\left(\theta^{\prime}\right) \hat{n} \cdot \nabla G\left(\theta ; \theta^{\prime}\right) d \ell\left(\theta^{\prime}\right) .
\end{aligned}
$$

Hence, the total poloidal flux of the vacuum equilibrium field is given by the sum of the flux produced by $K_{T}$ flowing on the plasma boundary $\ell_{P}(a)$ and the flux produced by the external coils:

$$
\begin{aligned}
\Phi(\vec{r}) & =\Phi_{\text {self }}(\vec{r})+\Phi_{\text {ext }}(\vec{r}) \\
& =\mu_{0} \oint_{\ell_{P}(a)} K_{T}\left(\theta^{\prime}\right) G\left(\vec{r} ; \theta^{\prime}\right) d \ell\left(\theta^{\prime}\right)+\mu_{0} \sum_{k} I_{k} G\left(\vec{r} ; \vec{r}_{k}\right) .
\end{aligned}
$$

Near the plasma boundary, the last term approximates the flux produced by the surface current $K_{T \text {,ext }}$ flowing on the contour $\ell$.

Using the expression for the equivalent surface current density on the internal flux surfaces

$$
\begin{aligned}
K_{T}(\rho, \theta) & =\frac{\hat{n} \cdot \nabla \Phi_{P}}{2 \pi \mu_{0} h_{\zeta}}=\frac{|\nabla \rho|}{2 \pi \mu_{0} h_{\zeta}} \frac{d \Phi_{P}}{d \rho} \\
& =\frac{1}{\mu_{0}}\left(\frac{h_{\theta}(\rho, \theta)}{2 \pi \sqrt{g}(\rho, \theta)}\right) \frac{I_{T}(\rho)}{K(\rho)},
\end{aligned}
$$

the poloidal angle average $\left\langle\Phi_{\text {self }}(\theta)\right\rangle_{\theta}$ over the plasma boundary defines the external self-inductance of the plasma loop by the Faraday (flux linkage) method

$$
L_{e}(a)=\frac{1}{K(a)}\left\langle\left(\frac{h_{\theta}^{2}(a, \theta)}{\sqrt{g}(a, \theta)}\right)\left\langle G\left(\theta ; \theta^{\prime}\right)\right\rangle_{\theta^{\prime}}\right\rangle_{\theta},
$$

where (cf. Sec. IV in Paper I)

$$
K(a)=\frac{1}{\mu_{0}}\left\langle\frac{h_{\theta}^{2}(a, \theta)}{\sqrt{g}(a, \theta)}\right\rangle_{\theta} .
$$

Therefore, the value of the total poloidal flux at the plasma boundary is given by

$$
\begin{aligned}
\Phi(a)= & L_{e}(a) I_{T}(a)=\frac{I_{T}(a)}{K(a)}\left\langle\left(\frac{h_{\theta}^{2}\left(a, \theta^{\prime}\right)}{\sqrt{g}\left(a, \theta^{\prime}\right)}\right) G\left(\theta ; \theta^{\prime}\right)\right\rangle_{\theta^{\prime}} \\
& +\mu_{0} \sum_{k} I_{k} G\left(\theta ; \vec{r}_{k}\right) .
\end{aligned}
$$

In order to eliminate superconducting solutions, which do not change the equilibrium field inside the contour $\ell$, it is assumed that

$$
\sum_{k}\left\langle\Phi_{k}(\theta)\right\rangle_{\theta}=\mu_{0} \sum_{k} I_{k}\left\langle G\left(\theta ; \vec{r}_{k}\right)\right\rangle_{\theta}=0
$$

The total magnetic flux $\Phi(a)$ on the plasma boundary is generated by the plasma current plus the currents in the external coil system. Since the plasma edge is a constant flux surface, the internal and external inductances can be calculated exactly by the method of flux linkages. The integration in all space used in the energy definition of the inductances is replaced by a surface integral intercepting all the magnetic flux of the system, the inductances being thereby characterized by the linkage of that flux with the currents in the system. Of course, the plasma boundary shape must be consistent with the external equilibrium field. Appendix A briefly reviews how to handle the Green's function singularity in the calculation of $L_{e}(a)$.

The total poloidal flux produced by the poloidal field coils of the ITER-like tokamak, shown schematically in Fig. 1 of Paper I, can be approximated by the sum of the flux produced by six thin cylindrical solenoids representing the segments of the central solenoid CS, and the flux produced by six circular loops representing the equilibrium field coil PF. This simplified geometry of the poloidal field coil system is presented in Table I, and is sufficient for the purpose of the present article. In general, the central solenoid is required to approximate an ideal transformer in the plasma region, that is, around the major axis $\left(R_{0}, 0\right)$ of the tokamak. Therefore, the currents are symmetrically distributed in the solenoid segments, with respect to the equatorial plane $Z=0$. Moreover, the currents are adjusted in order to cancel the dipole and quadrupole contributions of the central solenoid:
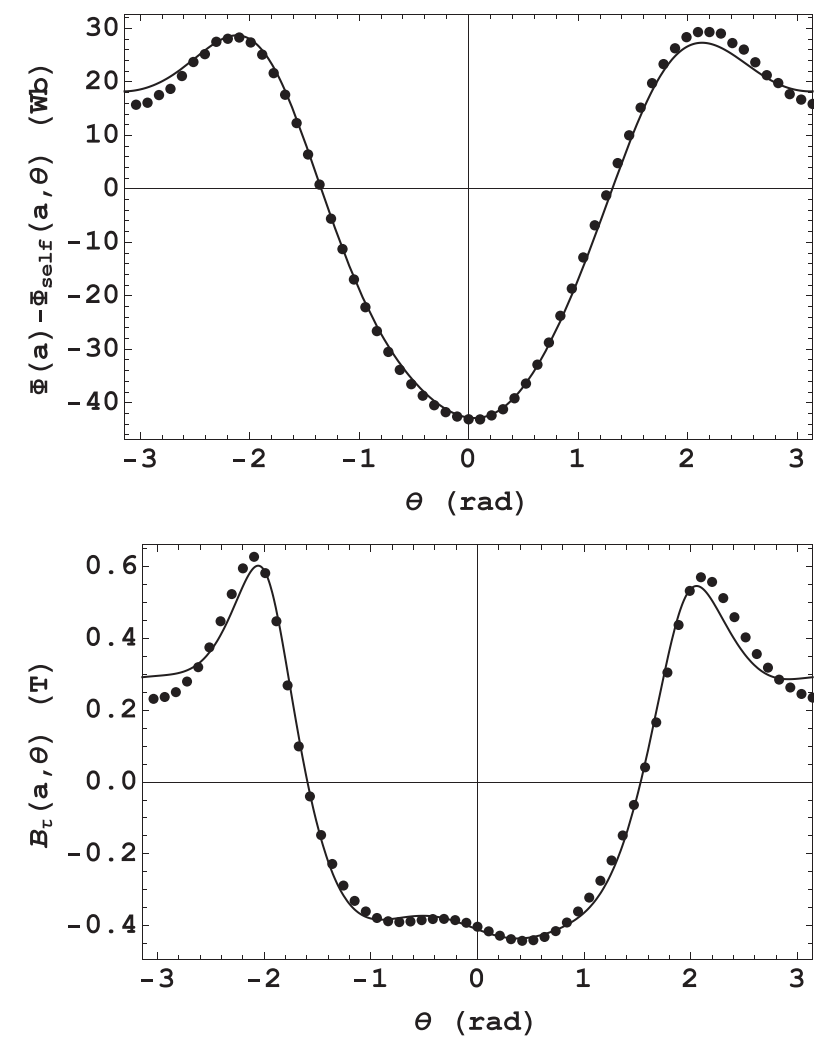

FIG. 1. Adjustment of currents in the poloidal field coils to fit the plasma self-flux difference (upper graph) and containing a magnetic field (lower graph). The dots show the sampled distributions of the poloidal flux difference and the containing field calculated from the equivalent surface current density on the plasma boundary, and the continuous lines show the corresponding distributions produced on the boundary by the poloidal field coils. 
TABLE I. Simplified geometry of the poloidal field coil system of the ITER-like tokamak.

\begin{tabular}{lcccccc}
\hline \hline $\begin{array}{l}\text { Central } \\
\text { solenoid }\end{array}$ & $R_{s}(\mathrm{~m})$ & $Z_{s}(\mathrm{~m})$ & $\Delta Z_{s}(\mathrm{~m})$ & $\begin{array}{c}\text { Equilibrium } \\
\text { field coils }\end{array}$ & $R_{k}(\mathrm{~m})$ & $Z_{k}(\mathrm{~m})$ \\
\hline CS1U & 1.677 & +1.014 & 1.977 & PF1 & 3.880 & +7.584 \\
CS1L & 1.677 & -1.014 & 1.977 & PF2 & 8.338 & +6.509 \\
CS2U & 1.677 & +3.041 & 1.977 & PF3 & 12.014 & +3.055 \\
CS2L & 1.677 & -3.041 & 1.977 & PF4 & 11.955 & -2.465 \\
CS3U & 1.677 & +4.974 & 1.789 & PF5 & 8.396 & -6.748 \\
CS3L & 1.677 & -4.974 & 1.789 & PF6 & 4.273 & -7.559 \\
\hline \hline
\end{tabular}

$$
\left\{\begin{array}{l}
\left.\sum_{s=1}^{6} \frac{\partial^{2}}{\partial R^{2}} \Phi_{s}\left(R, Z ; R_{s}, Z_{s}, \Delta Z_{s}\right)\right|_{R=R_{0}, Z=0}=0 \\
\left.\sum_{s=1}^{6} \frac{\partial^{4}}{\partial R^{4}} \Phi_{s}\left(R, Z ; R_{s}, Z_{s}, \Delta Z_{s}\right)\right|_{R=R_{0}, Z=0}=0
\end{array}\right.
$$

where $\Phi_{s}\left(R, Z ; R_{s}, Z_{s}, \Delta Z_{s}\right)$ is the poloidal flux produced by the segment $s$ of the central solenoid (cf. Appendix B). Hence, the currents in various segments are distributed in terms of, say, the current $I_{C S 1 U}$ in the segment CS1U as follows:

$$
\left\{\begin{array}{l}
I_{C S 1 L}=I_{C S 1 U}, \\
I_{C S 2 L}=I_{C S 2 U}=0.485194 I_{C S 1 U}, \\
I_{C S 3 L}=I_{C S 3 U}=1.89882 I_{C S 1 U} .
\end{array}\right.
$$

In this way, the poloidal flux produced by the central solenoid is uniform to order $\rho^{4}$ around the major axis $\left(R_{0}, 0\right)$.

Now, Table II lists the parameters of an internal equilibrium for the ITER-like tokamak shown in Fig. 6 of Paper I. The Dirichlet and Neumann parameters listed in this table completely define the plasma boundary and the equivalent surface current density distribution $K_{T}(\theta)$ on the boundary, according to the spectral representation of the internal flux

TABLE II. Parameters which describe the equilibrium of the ITER-like tokamak shown in Fig. 6 of Paper I. The plasma pressure and toroidal current profiles are defined as in Paper I.

\begin{tabular}{lcc}
\hline \hline Parameter set & \multicolumn{1}{c}{ Values } & \\
\hline Dirichlet & $R_{0}(a)=6.2 \mathrm{~m}$ & $Z_{0}(a)=0.550 \mathrm{~m}$ \\
& $a=2.000 \mathrm{~m}$ & $e(a)=1.757$ \\
& $S_{2}(a)=0.110$ & $A_{2}(a)=-0.00344$ \\
Neumann & $S_{3}(a)=-0.00735$ & $A_{3}(a)=-0.00817$ \\
& $R_{0}^{\prime}(a)=-0.0503$ & $Z_{0}^{\prime}(a)=-0.00117$ \\
& $e^{\prime}(a)=0.128 \mathrm{~m}^{-1}$ & \\
& $S_{2}^{\prime}(a)=0.0409 \mathrm{~m}^{-1}$ & $A_{2}^{\prime}(a)=-0.00161 \mathrm{~m}^{-1}$ \\
Magnetic axis & $S_{3}^{\prime}(a)=0.00495 \mathrm{~m}^{-1}$ & $A_{3}^{\prime}(a)=-0.00972 \mathrm{~m}^{-1}$ \\
& $R_{m}=6.240 \mathrm{~m}^{\prime}$ & $Z_{\mathrm{m}}=0.551 \mathrm{~m}$ \\
Matching radius & $\kappa_{m}=1.624$ & \\
Peaking factors & $\rho_{*}=0.577 \mathrm{~m}$ & \\
& $\alpha_{T}=0.7$ & $\alpha_{n}=\alpha_{T} / 2$ \\
Plasma current & $\alpha_{p}=\alpha_{n}+\alpha_{T}$ & $\alpha_{j}=3 \alpha_{T} / 2$ \\
Poloidal flux & $I_{T}(a)=15 \mathrm{MA}$ & $I_{P}(a)=9.78 \mathrm{MA}$ \\
Internal energy & $\Phi_{P}(a)=78.4 \mathrm{~Wb}$ & $\Phi(a)=106.9 \mathrm{~Wb}$ \\
Safety factors & $U(a)=731.6 \mathrm{MJ}$ & \\
\hline \hline
\end{tabular}

surfaces presented in Paper I. The spectral representation is reproduced below as applied to the ITER-like tokamak:

$$
\begin{aligned}
\frac{R(\rho, \theta)}{a}= & \frac{R_{0}(\rho)}{a}+\frac{\rho}{a} \cos \theta+\left(\frac{\rho}{a}\right)^{2}\left[S_{3}(\rho)(1-\cos \theta)\right. \\
& \left.+A_{3}(\rho) \sin \theta\right]-\left(\frac{\rho}{a}\right)\left[S_{2}(\rho)(1-\cos (2 \theta))\right. \\
& \left.-A_{2}(\rho) \sin (2 \theta)\right]-\left(\frac{\rho}{a}\right)^{2}\left[S_{3}(\rho)(1-\cos (3 \theta))\right. \\
& \left.-A_{3}(\rho) \sin (3 \theta)\right], \\
\frac{Z(\rho, \theta)}{e(\rho) a}= & \frac{Z_{0}(\rho)}{e(\rho) a}+\frac{\rho}{a} \sin \theta+\left(\frac{\rho}{a}\right)^{2}\left[A_{3}(\rho)(1-\cos \theta)\right. \\
& \left.-S_{3}(\rho) \sin \theta\right]-\left(\frac{\rho}{a}\right)\left[A_{2}(\rho)(1-\cos (2 \theta))\right. \\
& \left.+S_{2}(\rho) \sin (2 \theta)\right]-\left(\frac{\rho}{a}\right)^{2}\left[A_{3}(\rho)(1-\cos (3 \theta))\right. \\
& \left.+S_{3}(\rho) \sin (3 \theta)\right] .
\end{aligned}
$$

This representation includes the Shafranov shift, elongation, triangularity and quadrangularity effects, in an up-down asymmetric configuration.

For consistent equilibrium, the distributions of poloidal flux $\Phi_{\text {ext }}(\theta)$ and tangential magnetic field $B_{\tau, \text { ext }}(\theta)$ produced by external sources on the boundary, according to Eqs. (12) and (20), must satisfy the Dirichlet condition

$$
\begin{aligned}
\Phi_{\text {ext }}(\theta)= & \Phi(a)-\Phi_{\text {self }}(\theta)=L_{e}(a) I_{T}(a) \\
& -\mu_{0} \oint_{\ell_{P}(a)} K_{T}\left(\theta^{\prime}\right) G\left(\theta ; \theta^{\prime}\right) d \ell\left(\theta^{\prime}\right) \\
\cong & \mu_{0} \sum_{s=1}^{6} I_{s} G\left(\theta ; \vec{r}_{s}\right)+\mu_{0} \sum_{k=1}^{6} I_{k} G\left(\theta ; \vec{r}_{k}\right),
\end{aligned}
$$

and the Neumann condition

$$
\begin{aligned}
B_{\tau, \text { ext }}(\theta)= & \frac{\mu_{0}}{2 \pi h_{\zeta}(\theta)} \oint_{\ell_{P}(a)} K_{T}\left(\theta^{\prime}\right) \hat{n} \cdot \nabla G\left(\theta ; \theta^{\prime}\right) d \ell\left(\theta^{\prime}\right) \\
& -\frac{\mu_{0} K_{T}(\theta)}{2} \cong-\frac{\mu_{0}}{2 \pi h_{\zeta}(\theta)}\left(\sum_{s=1}^{6} I_{s} \hat{n} \cdot \nabla G\left(\theta ; \vec{r}_{s}\right)\right. \\
& \left.+\sum_{k=1}^{6} I_{k} \hat{n} \cdot \nabla G\left(\theta ; \vec{r}_{k}\right)\right) .
\end{aligned}
$$

The above Dirichlet and Neumann conditions (Cauchy condition) are not independent. They may be used separately or in a combined form to determine the currents in the poloidal field coils, $I_{s}$ and $I_{k}$, that best fit the equilibrium. When the containing field $B_{\tau, \text { ext }}(\theta)$ is adjusted separately (Neumann condition), one must take into account the superconducting constraint (32), which gives the poloidal angle average flux contribution of the central solenoid and equilibrium field coils on the plasma boundary: 


$$
\mu_{0} \sum_{s=1}^{6} I_{s}\left\langle G\left(\theta ; \vec{r}_{s}\right)\right\rangle_{\theta}+\mu_{0} \sum_{k=1}^{6} I_{k}\left\langle G\left(\theta ; \vec{r}_{k}\right)\right\rangle_{\theta}=0 .
$$

This constraint is properly taken into account when adjusting the poloidal flux $\Phi_{\text {ext }}(\theta)$ (Dirichlet condition), which explicitly gives the external inductance contribution of the plasma. In the present application, there are seven independent external currents that can be adjusted, and the different approaches give similar, though not identical results. Adjusting the Neumann and superconducting conditions only (this adjustment has a better quality), the equilibrium listed in Table II can be fitted with the following values of the coil currents:

$$
\left\{\begin{array}{l}
I_{C S 1 U}=I_{C S 1 L}=5.674 \mathrm{MA}, \\
I_{C S 2 U}=I_{C S 2 L}=2.753 \mathrm{MA}, \\
I_{C S 3 U}=I_{C S 3 L}=10.77 \mathrm{MA}, \\
I_{P F 1}=16.38 \mathrm{MA}, \quad I_{P F 2}=-3.802 \mathrm{MA}, \\
I_{P F 3}=-3.744 \mathrm{MA}, \quad I_{P F 4}=0.2406 \mathrm{MA}, \\
I_{P F 5}=-14.53 \mathrm{MA}, \quad I_{P F 6}=34.37 \mathrm{MA} .
\end{array}\right.
$$

The adjustment prescribed by Eqs. (36) and (37), taken separately or in a combined form [with the possible addition of constraints given by Eqs. (32) and (34)] is quite robust for a reasonable number of coils, and can be carried out starting with proper signs and order of magnitude values of the coil currents. The above adjustment, for example, started with $I_{P F 1}=I_{P F 6}=10^{7} \mathrm{MA}$ and $I_{P F 2}=I_{P F 3}=I_{P F 4}=I_{P F 5}$ $=-10^{7} \mathrm{MA}$ (note that even the sign of $I_{P F 4}$ changed during the adjustment procedure). Of course, the tokamak designer must have some feeling for the effect of each coil in the plasma equilibrium.

Figure 1 shows the adjustment of the boundary conditions (36) and (37) obtained with the above values of the currents in the poloidal field coils. The figure shows the sampled distributions, used in the adjustment, both for the plasma self-flux difference $\Phi(a)-\Phi_{\text {self }}(\theta)$ and for the containing field $B_{\tau}(\theta)$ calculated from the equivalent surface current $K_{T}(\theta)$. In the present article, a likely excessive number of sampling points were used, as shown in Fig. 1, but this depends on the variation of $\Phi_{\text {self }}(\theta)$ and $B_{\tau}(\theta)$ along the boundary (the variation is larger for a low aspect ratio tokamak). Also, the figure shows the poloidal flux $\Phi_{\text {ext }}(\theta)$ and the tangential component of the magnetic field $B_{\tau, \text { ext }}(\theta)$ produced on the plasma boundary by the poloidal field coils. A better fit is obtained if the currents in the twelve poloidal field coils are independently adjusted, i.e., without using condition (34), but this does not constitute a practical solution.

In this section, it was shown how to adjust the currents in the poloidal field coils of a tokamak configuration to approximately obtain a desired plasma boundary shape. This procedure is part of the tokamak design. However, due to the finite number of poloidal field coils, this adjustment may not be completely satisfactory, as shown in Fig. 1. In Sec. III, the adjustment of the plasma boundary to the prescribed external field is considered, which constitutes the properly named free-boundary equilibrium problem.

\section{PLASMA BOUNDARY ADJUSTMENT}

The plasma boundary must conform to the boundary conditions (36) and (37) imposed by the poloidal field coils. For this purpose, the external equilibrium problem can be reduced to a system of equations by taking moments of Eq. (26). Defining the Neumann coefficients at the plasma boundary for the external coils

$$
\left\{\begin{array}{l}
N_{k, n}^{(s)}=\left\langle\hat{n} \cdot \nabla G\left(\theta ; \vec{r}_{k}\right) \cos n \theta\right\rangle_{\theta} \\
N_{k, n}^{(a)}=\left\langle\hat{n} \cdot \nabla G\left(\theta ; \vec{r}_{k}\right) \sin n \theta\right\rangle_{\theta},
\end{array}\right.
$$

where $n=0,1,2 \ldots$, one obtains a system of symmetric and antisymmetric badly conditioned linear moment equations for the coil currents:

$$
\left\{\begin{array}{l}
\sum_{k} N_{k, n}^{(s)} I_{k}=\pi\left\langle h_{\zeta}(\theta) K_{T}(\theta) \cos n \theta\right\rangle_{\theta}-2 \pi\left\langle\left\langle h_{\theta}\left(\theta^{\prime}\right) K_{T}\left(\theta^{\prime}\right) \hat{n} \cdot \nabla G\left(\theta ; \theta^{\prime}\right)\right\rangle_{\theta^{\prime}} \cos n \theta\right\rangle_{\theta} \\
\sum_{k} N_{k, n}^{(a)} I_{k}=\pi\left\langle h_{\zeta}(\theta) K_{T}(\theta) \sin n \theta\right\rangle_{\theta}-2 \pi\left\langle\left\langle h_{\theta}\left(\theta^{\prime}\right) K_{T}\left(\theta^{\prime}\right) \hat{n} \cdot \nabla G\left(\theta ; \theta^{\prime}\right)\right\rangle_{\theta^{\prime}} \sin n \theta\right\rangle_{\theta}
\end{array}\right.
$$

The coil currents, which must satisfy the additional constraint (32), can be determined by a least-squares fitting procedure based on these moment equations. This gives nearly the same values for the adjusted coil currents, as determined in Sec. II. However, the objective of the present section is to find the equivalent surface current density $K_{T}(\theta)$ that approximately satisfies the moment equations.

Introducing the symmetric, $N_{n}^{(s)}$, and antisymmetric, $N_{n}^{(a)}$, Neumann coefficients of order $n$ for the plasma boundary, the above equations can be written in the simplified form

$$
\left\{\begin{array}{l}
\sum_{k} N_{k, n}^{(s)} I_{k}=N_{n}^{(s)} I_{T}(a) \\
\sum_{k} N_{k, n}^{(a)} I_{k}=N_{n}^{(a)} I_{T}(a)
\end{array}\right.
$$

The Neumann coefficients were introduced in a different context in Ref. 12, where it was shown how to handle the singularity of the normal component of Green's function gradient in the left-hand side of the above equations. This procedure is reviewed in Appendix B. 
The superconducting constraint (32) and the Neumann moment Eqs. (42) may be combined in a norm that quantifies the boundary condition mismatch between the plasma and the simplified poloidal field coil system of the ITER-like tokamak:

$$
\begin{aligned}
\text { Norm }= & \left\{\left(\sum_{s=1}^{6} \frac{\left\langle\Phi_{s}(\theta)\right\rangle_{\theta}}{\mu_{0} R_{0}(a) I_{T}(a)}+\sum_{k=1}^{6} \frac{\left\langle\Phi_{k}(\theta)\right\rangle_{\theta}}{\mu_{0} R_{0}(a) I_{T}(a)}\right)^{2}\right. \\
& +\sum_{n=1}^{6}\left[\left(N_{n}^{(s)}-\sum_{s=1}^{6} N_{s, n}^{(s)} \frac{I_{s}}{I_{T}(a)}-\sum_{k=1}^{6} N_{k, n}^{(s)} \frac{I_{k}}{I_{T}(a)}\right)^{2}\right. \\
& \left.\left.+\left(N_{n}^{(a)}-\sum_{s=1}^{6} N_{s, n}^{(a)} \frac{I_{s}}{I_{T}(a)}-\sum_{k}^{6} N_{k, n}^{(a)} \frac{I_{k}}{I_{T}(a)}\right)^{2}\right]\right\}^{1 / 2} .
\end{aligned}
$$

The currents $I_{s}$ and $I_{k}$ that best fit the equilibrium listed in Table II can be obtained searching for a minimum of the norm, resulting in values similar to the ones obtained in Sec. II. Conversely, for fixed current values, the plasma boundary parameters can be adjusted to satisfy the combined conditions (36) and (37), searching again for a minimum of the norm. The boundary is characterized by the Dirichlet parameters $a, R_{0}(a), Z_{0}(a), e(a), S_{2}(a), A_{2}(a), S_{3}(a), A_{3}(a)$, which specify the plasma shape, and the Neumann parameters $R_{0}^{\prime}(a), Z_{0}^{\prime}(a), e^{\prime}(a), S_{2}^{\prime}(a), A_{2}^{\prime}(a), S_{3}^{\prime}(a), A_{3}^{\prime}(a)$, which determine the radial derivatives of the Fourier coefficients at the plasma boundary (cf. Sec. V of Paper I). Note that the flux and current values in norm (43) are normalized in terms of the major radius $R_{0}(a)$ and the plasma current $I_{T}(a)$. The Neumann coefficients correspond to gradients completely normalized by $R_{0}(a)$ as well. Therefore, similar plasma shapes can be obtained scaling the geometry and/or the currents (the coil geometry must be normalized accordingly). The values of $R_{0}(a)$ and $I_{T}(a)$, plus the coil positions and currents, are input into the minimization procedure.

Figure 2 shows the decrease in the norm for each iteration step in a minimization procedure using Newton's method. The norm rapidly decreases by more than two orders of magnitude, but the final result depends on the numerical

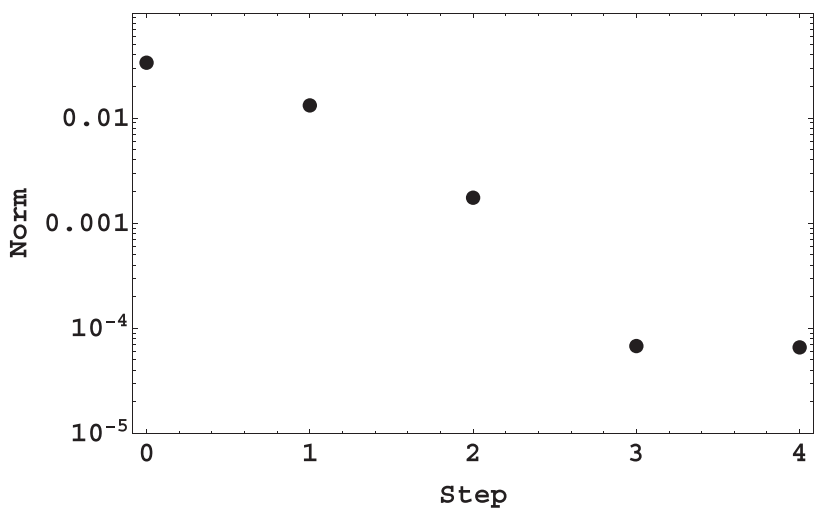

FIG. 2. Adjustment of the Cauchy condition on the plasma boundary to the external sources. The figure shows the decrease in the norm of the poloidal flux and Neumann moment mismatch, between plasma and poloidal field coils, for each iteration step. precision of the calculations. Table III lists the adjusted Dirichlet and Neumann parameters for an ITER-like equilibrium consistent with the values of the currents fixed in Sec. II for the poloidal field coils, and Fig. 3 shows the adjustment obtained in the Cauchy condition at the plasma boundary. This result can be compared with the relatively large mismatch in the Cauchy condition shown in Fig. 1 for the initial, non-adjusted plasma boundary. Finally, Fig. 4 compares the distribution of the equivalent surface current density on the plasma boundary for the initial and adjusted Cauchy conditions. This comparison indicates that the internal equilibrium solution must also be modified to conform to the boundary conditions imposed by the poloidal field coils, a problem that is treated in Sec. IV.

\section{INTERNAL EQUILIBRIUM FIELD ADJUSTMENT}

In Sec. III, the equivalent surface current density $K_{T}(\theta)$ on the plasma boundary was adjusted to satisfy the Cauchy boundary condition imposed by the poloidal field coils. According to the integral consistency condition (8), the surface current density completely specifies the internal current density distribution. This "inverse" problem can be solved in an approximate form only, within the order of the spectral representation given by Eq. (35), using the methods presented in Paper I.

The Fourier coefficients of the spectral representation are described by piecewise continuous approximations as discussed in Sec. V, Paper I. However, extra degrees of freedom must be added to the description of the internal flux surfaces in order to satisfy both Dirichlet and Neumann boundary conditions. For this purpose, extended piecewise continuous approximations are constructed matching the Taylor series in the plasma core with cubic series expansions

TABLE III. Parameters which describe the equilibrium of the ITER-like

\begin{tabular}{|c|c|c|}
\hline Parameter set & Values & \\
\hline Dirichlet & $\begin{array}{c}R_{0}(a)=6.2 \mathrm{~m} \\
a=1.984 \mathrm{~m} \\
S_{2}(a)=0.118 \\
S_{3}(a)=0.0185\end{array}$ & $\begin{array}{c}Z_{0}(a)=0.630 \mathrm{~m} \\
e(a)=1.724 \\
A_{2}(a)=0.00506 \\
A_{3}(a)=-0.00584\end{array}$ \\
\hline Neumann & $\begin{array}{c}R_{0}^{\prime}(a)=-0.129 \\
e^{\prime}(a)=0.0378 \mathrm{~m}^{-1} \\
S_{2}^{\prime}(a)=-0.0313 \mathrm{~m}^{-1} \\
S_{3}^{\prime}(a)=-0.0292 \mathrm{~m}^{-1}\end{array}$ & $\begin{array}{l}A_{2}^{\prime}(a)=-0.0192 \mathrm{~m}^{-1} \\
A_{3}^{\prime}(a)=0.00114 \mathrm{~m}^{-1}\end{array}$ \\
\hline Magnetic axis & $\begin{array}{c}R_{m}=6.277 \mathrm{~m} \\
\kappa_{m}=1.542\end{array}$ & $Z_{\mathrm{m}}=0.639 \mathrm{~m}$ \\
\hline Matching coefficients & $\begin{aligned} R_{0}^{\prime \prime}(a) & =-0.0311 \mathrm{~m}^{-1} \\
e^{\prime \prime}(a) & =-0.549 \mathrm{~m}^{-2} \\
S_{2}^{\prime \prime}(a) & =-0.236 \mathrm{~m}^{-2} \\
S_{3}^{\prime \prime}(a) & =-0.272 \mathrm{~m}^{-2}\end{aligned}$ & $\begin{array}{c}Z_{0}^{\prime \prime}(a)=-0.213 \mathrm{~m}^{-1} \\
A_{2}^{\prime \prime}(a)=-0.0678 \mathrm{~m}^{-2} \\
A_{3}^{\prime \prime}(a)=0.0257 \mathrm{~m}^{-2}\end{array}$ \\
\hline Matching radius & $\rho_{*}=1.274 \mathrm{~m}$ & \\
\hline Peaking factors & $\begin{array}{c}\alpha_{T}=0.643 \\
\alpha_{p}=\alpha_{n}+\alpha_{T}\end{array}$ & $\begin{array}{c}\alpha_{n}=\alpha_{T} / 2 \\
\alpha_{j}=3 \alpha_{T} / 2\end{array}$ \\
\hline Plasma current & $I_{T}(a)=15 \mathrm{MA}$ & $I_{P}(a)=10.4 \mathrm{MA}$ \\
\hline Poloidal flux & $\Phi_{P}(a)=76.4 \mathrm{~Wb}$ & $\Phi(a)=110.2 \mathrm{~Wb}$ \\
\hline Internal energy & $U(a)=716.1 \mathrm{MJ}$ & \\
\hline Safety factors & $q(0)=1.000$ & $q(a)=2.744$ \\
\hline
\end{tabular}
tokamak with the adjusted Cauchy condition shown in Fig. 5. The plasma pressure and toroidal current profiles are defined as in Paper I. 

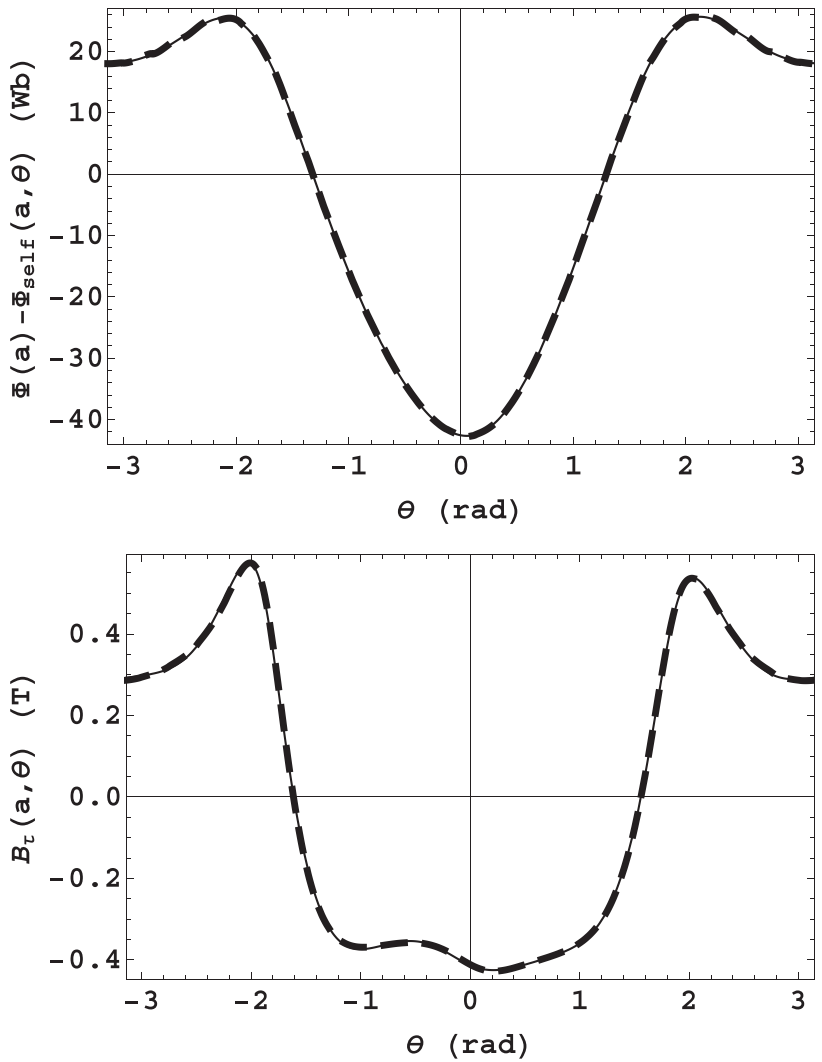

FIG. 3. Adjusted poloidal flux (upper graph) and tangential component of the magnetic field (lower graph) at the plasma boundary. The thick dashed lines show the poloidal flux difference and the containing field calculated from the equivalent surface current density on the plasma boundary, and the thin continuous lines show the distributions produced by the poloidal field coils.

at the plasma border, as illustrated for the position of the major radius $R_{0}(\rho)$ :

$$
\begin{aligned}
R_{0, \text { core }}(\rho)= & R_{m}+\frac{\rho^{2}}{2} R_{0}^{\prime \prime}(0)+\frac{\rho^{4}}{24} R_{0}^{(4)}(0)+\cdots \quad 0 \leqslant \rho \leqslant \rho_{*}, \\
R_{0, \text { edge }}(\rho)= & R_{0}(a)-(a-\rho) R_{0}^{\prime}(a)+\frac{(a-\rho)^{2}}{2} R_{0}^{\prime \prime}(a) \\
& -\frac{(a-\rho)^{3}}{6} R_{0}^{(3)}(a) \cdots \quad \rho_{*} \leqslant \rho \leqslant a .
\end{aligned}
$$

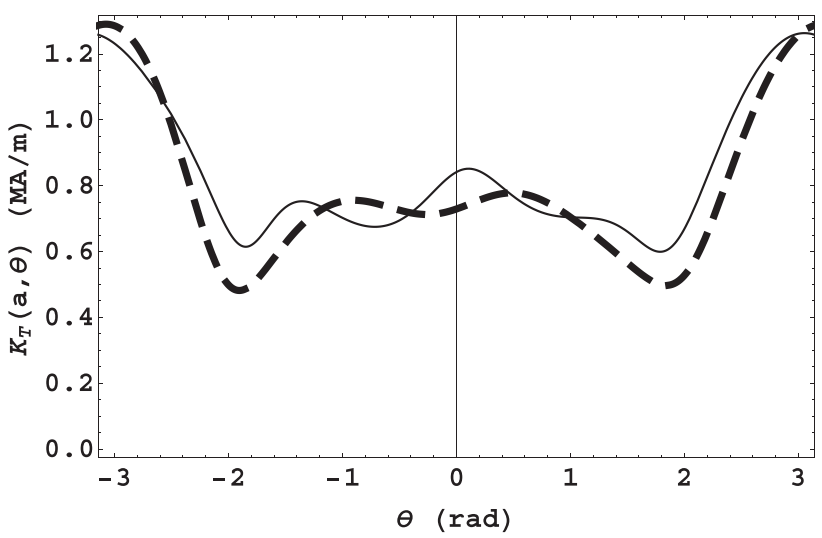

FIG. 4. Comparison between the equivalent surface current densities on the plasma boundary defined by either the initial (thick dashed line) or adjusted (thin continuous line) Cauchy conditions.
In accordance with Paper I, a matching radius $\rho_{*}$ is defined such that

$$
\begin{aligned}
& R_{0, \text { core }}\left(\rho_{*}\right)=R_{0, \text { edge }}\left(\rho_{*}\right), \quad R_{0, \text { core }}^{\prime}\left(\rho_{*}\right)=R_{0, \text { edge }}^{\prime}\left(\rho_{*}\right), \\
& R_{0, \text { core }}^{\prime \prime}\left(\rho_{*}\right)=R_{0, \text { edge }}^{\prime \prime}\left(\rho_{*}\right),
\end{aligned}
$$

leading to

$$
\begin{aligned}
R_{0}^{\prime \prime}(0)= & {\left[-12\left(3 a-\rho_{*}\right)\left(R_{m}-R_{0}(a)\right)-6\left(2 a-\rho_{*}\right)^{2} R_{0}^{\prime}(a)\right.} \\
& \left.+\left(a-\rho_{*}\right)\left(6 a^{2}-4 a \rho_{*}+\rho_{*}^{2}\right) R_{0}^{\prime \prime}(a)\right] /\left[\left(8 a^{2}-a \rho_{*}\right.\right. \\
& \left.\left.-\rho_{*}^{2}\right) \rho_{*}\right], \\
R_{0}^{(4)}(0)= & {\left[72\left(a+\rho_{*}\right)\left(R_{m}-R_{0}(a)\right)+24\left(2 a^{2}+2 a \rho_{*}-\rho_{*}^{2}\right)\right.} \\
& \left.\times R_{0}^{\prime}(a)-12 a\left(a-\rho_{*}\right)\left(a+2 \rho_{*}\right) R_{0}^{\prime \prime}(a)\right] /\left[\left(8 a^{2}-a \rho_{*}\right.\right. \\
& \left.\left.-\rho_{*}^{2}\right) \rho_{*}^{3}\right], \\
R_{0}^{(3)}(a)= & -6\left[8\left(R_{m}-R_{0}(a)\right)+\left(8 a-3 \rho_{*}\right) R_{0}^{\prime}(a)\right. \\
& \left.-a\left(4 a-3 \rho_{*}\right) R_{0}^{\prime \prime}(a)\right] /\left[\left(a-\rho_{*}\right)\left(8 a^{2}-a \rho_{*}-\rho_{*}^{2}\right)\right],
\end{aligned}
$$

with similar expressions for $Z_{0}(\rho)$ and $e(\rho)$. Likewise, the extended piecewise continuous approximation for the symmetric triangularity coefficient is:

$$
\begin{aligned}
S_{2, \text { core }}(\rho)= & \rho S_{2}^{\prime}(0)+\frac{\rho^{3}}{6} S_{2}^{(3)}(0)+\cdots \quad 0 \leqslant \rho \leqslant \rho_{*}, \\
S_{2, \text { edge }}(\rho)= & S_{2}(a)-(a-\rho) S_{2}^{\prime}(a)+\frac{(a-\rho)^{2}}{2} S_{2}^{\prime \prime}(a) \\
& -\frac{(a-\rho)^{3}}{6} S_{2}^{(3)}(a) \cdots \quad \rho_{*} \leqslant \rho \leqslant a .
\end{aligned}
$$

Using the same matching radius $\rho_{*}$, such that

$S_{2, \text { core }}\left(\rho_{*}\right)=S_{2, \text { edge }}\left(\rho_{*}\right), \quad S_{2, \text { core }}^{\prime}\left(\rho_{*}\right)=S_{2, \text { edge }}^{\prime}\left(\rho_{*}\right), \quad$ and

$S_{2, \text { core }}^{\prime \prime}\left(\rho_{*}\right)=S_{2, \text { edge }}^{\prime \prime}\left(\rho_{*}\right)$,

one obtains

$$
\begin{aligned}
S_{2}^{\prime}(0) & =\frac{6 S_{2}(a)-2\left(2 a-\rho_{*}\right) S_{2}^{\prime}(a)+a\left(a-\rho_{*}\right) S_{2}^{\prime \prime}(a)}{2\left(a+\rho_{*}\right)}, \\
S_{2}^{(3)}(0) & =\frac{-6 S_{2}(a)+6 a S_{2}^{\prime}(a)-\left(2 a^{2}-a \rho_{*}-\rho_{*}^{2}\right) S_{2}^{\prime \prime}(a)}{a\left(a+\rho_{*}\right) \rho_{*}}, \\
S_{2}^{(3)}(a) & =\frac{6 S_{2}(a)-6 a S_{2}^{\prime}(a)+\left(3 a^{2}-\rho_{*}^{2}\right) S_{2}^{\prime \prime}(a)}{a\left(a^{2}-\rho_{*}^{2}\right)}
\end{aligned}
$$

with similar expressions for $A_{2}(\rho), S_{3}(\rho)$ and $A_{3}(\rho)$. These cubic approximations for the plasma edge are reduced to the quadratic approximations used in Paper I by taking

$$
\begin{aligned}
& R_{0}^{\prime \prime}(a)=\frac{8\left(R_{m}-R_{0}(a)\right)+\left(8 a-3 \rho_{*}\right) R_{0}^{\prime}(a)}{a\left(4 a-3 \rho_{*}\right)}, \\
& S_{2}^{\prime \prime}(a)=-\frac{6\left(S_{2}(a)-a S_{2}^{\prime}(a)\right)}{3 a^{2}-\rho_{*}^{2}},
\end{aligned}
$$

with similar expressions for $Z_{0}^{\prime \prime}(a), e^{\prime \prime}(a), A_{2}^{\prime \prime}(a), S_{3}^{\prime \prime}(a)$ and $A_{3}^{\prime \prime}(a)$. 
The extended piecewise continuous approximations define the flux surface geometry in terms of: the magnetic axis parameters, $R_{m}, Z_{m}$, and $\kappa_{m}$; the shape of the plasma, $a$, $R_{0}(a), \quad Z_{0}(a), e(a), S_{2}(a), \quad A_{2}(a), S_{3}(a), A_{3}(a)$ (Dirichlet parameters); the first order radial derivatives at the plasma boundary, $R_{0}^{\prime}(a), Z_{0}^{\prime}(a), e^{\prime}(a), S_{2}^{\prime}(a), A_{2}^{\prime}(a), S_{3}^{\prime}(a), A_{3}^{\prime}(a)$ (Neumann parameters); the second order radial derivatives at the plasma boundary, $R_{0}^{\prime \prime}(a), Z_{0}^{\prime \prime}(a), e^{\prime \prime}(a), S_{2}^{\prime \prime}(a), A_{2}^{\prime \prime}(a)$, $S_{3}^{\prime \prime}(a), A_{3}^{\prime \prime}(a)$ (matching coefficients); and the matching radius, $\rho_{*}$. The Dirichlet and Neumann parameters are defined by the adjusted equivalent surface current density $K_{T}(\theta)$. Therefore, the magnetic axis parameters and the set of second order radial derivatives (matching coefficients) can be obtained from Paper I, by solving Eqs. (76)-(78) coupled with Eqs. (C8)-(C14) of Paper I. There are ten equations available for ten variables, with the optimum matching radius $\rho_{*}$ being determined searching for a stationary value of internal energy $U(a)$ given in Sec. IV of Paper I by Eq. (58). Table III lists the parameters of the finally adjusted ITER-like equilibrium, consistent with the Cauchy condition imposed by the poloidal field coils.

Figure 5 shows the internal flux surfaces for the adjusted equilibrium, and Fig. 6 shows the radial profiles of the plasma poloidal flux $\Phi_{P}(\rho)$ and of the Fourier amplitudes. Since a small decrease of the plasma poloidal cross-section has been imposed by the external field, an equivalent increase in the toroidal current density results in a safety factor below one on the magnetic axis for an initial peaking factor $\alpha_{T}=0.7$. This can be compensated, as shown in Table III, by adopting slightly flatter profiles with $\alpha_{T}=0.643$. Figure 7 shows the profiles of the toroidal and poloidal current densities along the equatorial plane, for the ITER-like tokamak equilibrium shown in Figs. 5, and Fig. 8 shows the respective distribution of the equivalent surface current density in the internal flux surfaces. Finally, Fig. 9 shows a contour plot of the external equilibrium field and the internal flux surfaces for the consistent ITER-like equilibrium listed in Table III. This figure shows that equilibrium has been attained with open field lines adjusted to the lower divertor geometry. Moreover, between the outer border of the plasma and the limiter center line, indicated by the thin black line in Figs. 5 and 9, there is a gap of about $9.7 \mathrm{~cm}$.

\section{APPLICATION TO THE ITER EQUILIBRIUM AT BURN}

In this section, the model is applied to the ITER equilibrium at the start of the burn magnetic configuration. The equilibrium currents in the poloidal field coil system of the ITER burn configuration are given in Ref. 13 and reproduced in the following table:

$$
\begin{cases}I_{C S 1 U}=-20.09 \mathrm{MA}, & I_{C S 1 L}=-20.09 \mathrm{MA}, \\ I_{C S 2 U}=-9.68 \mathrm{MA}, & I_{C S 2 L}=-9.50 \mathrm{MA}, \\ I_{C S 3 U}=-0.40 \mathrm{MA}, & I_{C S 3 L}=3.22 \mathrm{MA}, \\ I_{P F 1}=4.91 \mathrm{MA}, & I_{P F 2}=-2.04 \mathrm{MA}, \\ I_{P F 3}=-6.52 \mathrm{MA}, & I_{P F 4}=-4.69 \mathrm{MA}, \\ I_{P F 5}=-7.54 \mathrm{MA}, & I_{P F 6}=17.20 \mathrm{MA} .\end{cases}
$$

Although the geometry of the poloidal field coils in the reference design ${ }^{13}$ is slightly different from that used in the present article, the simple current geometry and the initial positions listed in Table I will be maintained. In general, the geometry of the poloidal field coil system, in particular, of the extreme coils which form the central solenoid, affects the plasma shape. If necessary, improved representations can be obtained, both for the central solenoid and the external poloidal field coils, by the superposition of various thin solenoids or current loops. Presumably, the current distribution of the above reference coils was adjusted according to a minimum energy criterion, with all currents taken independently. Since the reference design includes a superconducting surface current distribution $\left(\left\langle\Phi_{\text {ext }}(\theta)\right\rangle_{\theta} \neq 0\right)$, the plasma boundary conveniently conformed with the external field using the Dirichlet condition (12):

$$
\Phi_{\text {self }}(\theta)+\Phi_{\text {ext }}(\theta)=\Phi(a),
$$

where
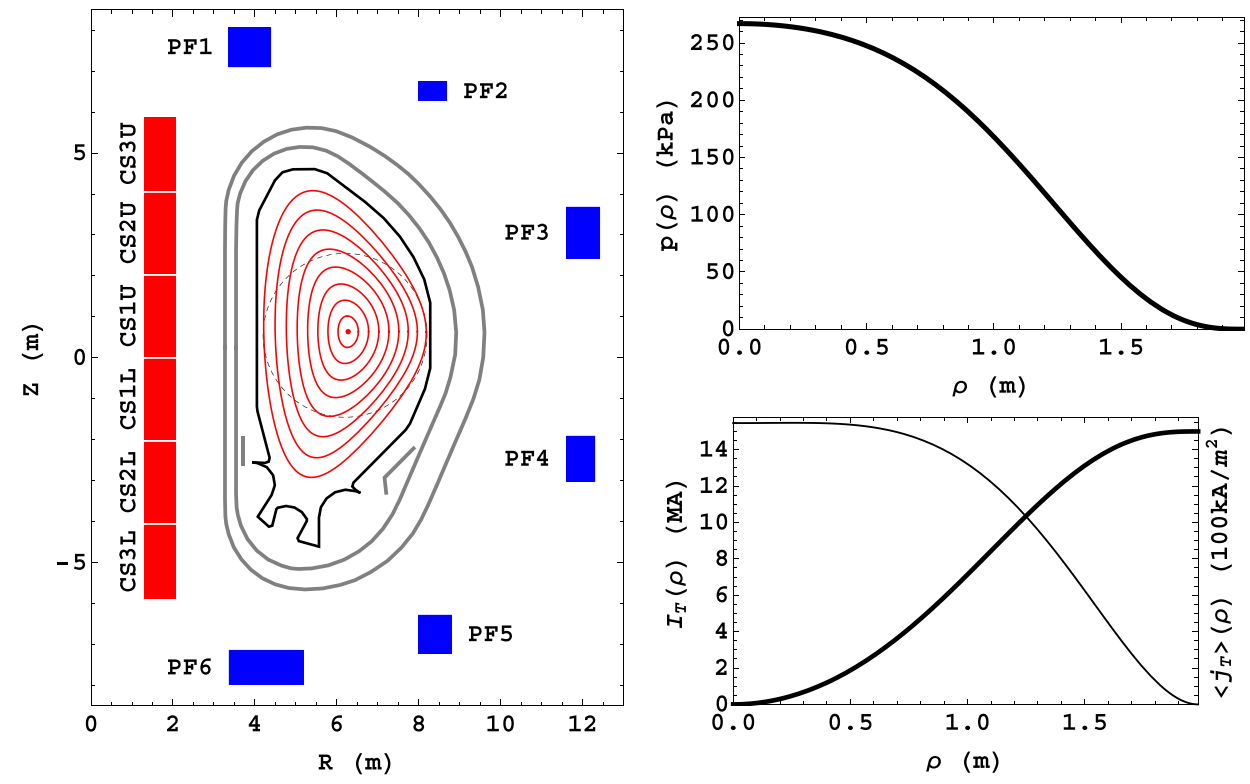

FIG. 5. Left: flux surface geometry inside the ITER-like tokamak plasma for the equilibrium with the adjusted Cauchy condition. The plasma is surrounded by the limiter and vacuum vessel walls; the rectangles represent the poloidal cross-sections of the central solenoid (CS) and equilibrium field coils (PF). Right: profiles of the plasma pressure, $p(\rho)$, toroidal current, $I_{T}(\rho)$ (thick line), and approximate toroidal current density, $\left\langle j_{T}\right\rangle(\rho)$ (thin line). 

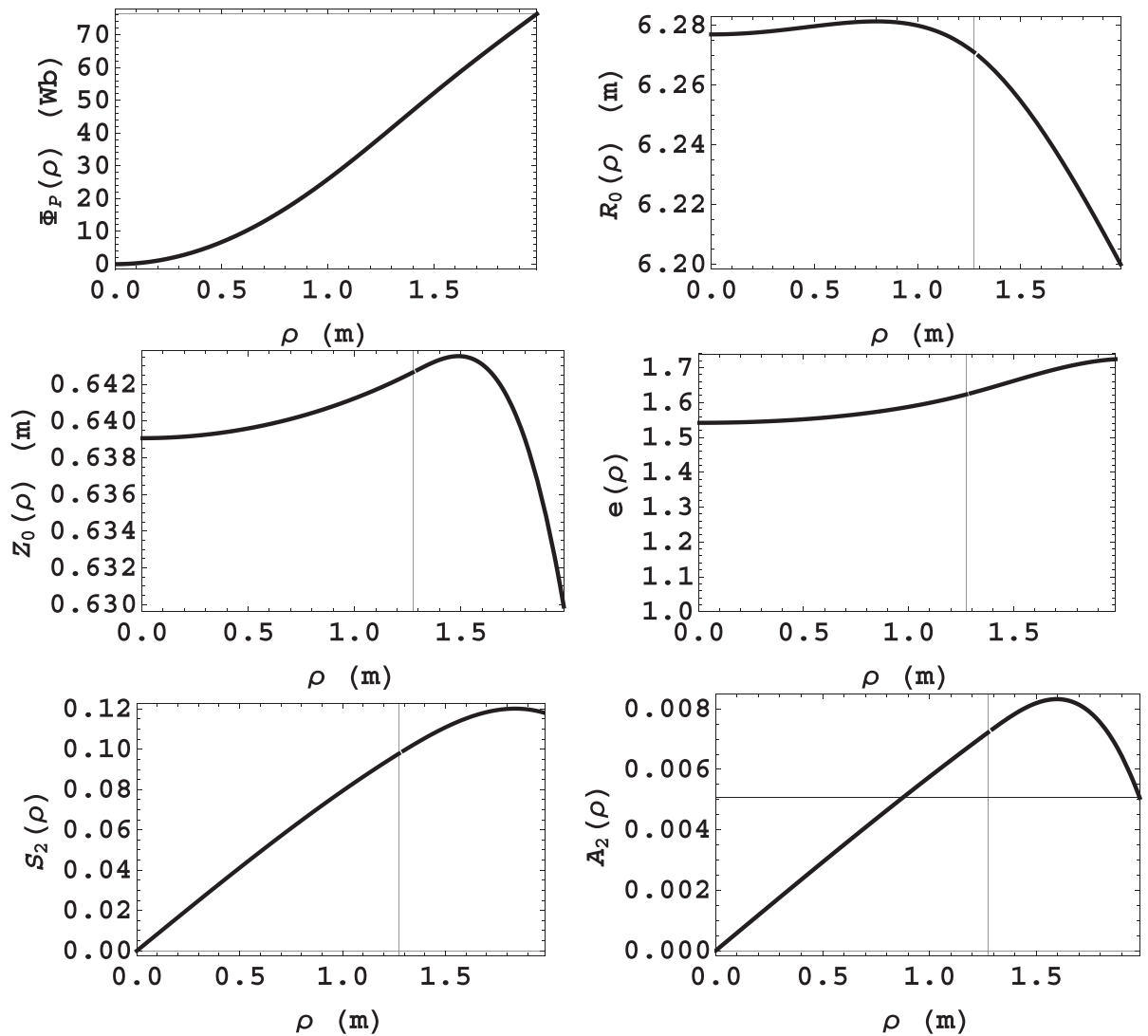

FIG. 6. Profiles of the poloidal flux, $\Phi_{P}(\rho)$; the main Fourier amplitudes, $R_{0}(\rho), Z_{0}(\rho)$ and $e(\rho)$; the symmetric, $S_{2}(\rho)$, and antisymmetric, $A_{2}(\rho)$, triangularity coefficients; and the symmetric, $S_{3}(\rho)$, and antisymmetric, $A_{3}(\rho)$, quadrangularity coefficients for the ITER-like equilibrium shown in Fig. 5. The thin vertical line indicates the position of the matching radius, $\rho_{*}$
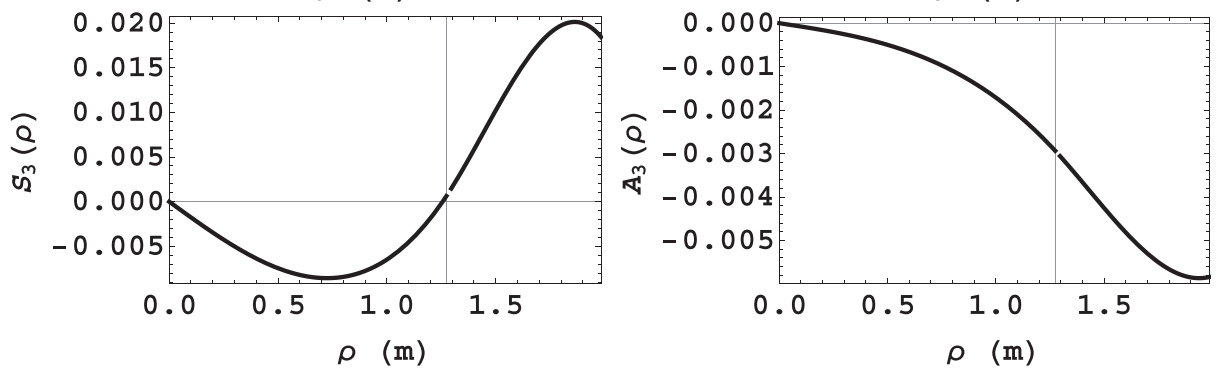

$\Phi_{\text {self }}(\theta)=2 \pi \mu_{0}\left\langle h_{\theta}\left(\theta^{\prime}\right) K_{T}\left(\theta^{\prime}\right) G\left(\theta ; \theta^{\prime}\right)\right\rangle_{\theta^{\prime}}$,

$\Phi_{\text {ext }}(\theta)=\mu_{0} \sum_{s=1}^{6} I_{s} G\left(\theta ; \vec{r}_{s}\right)+\mu_{0} \sum_{k=1}^{6} I_{k} G\left(\theta ; \vec{r}_{k}\right)$.

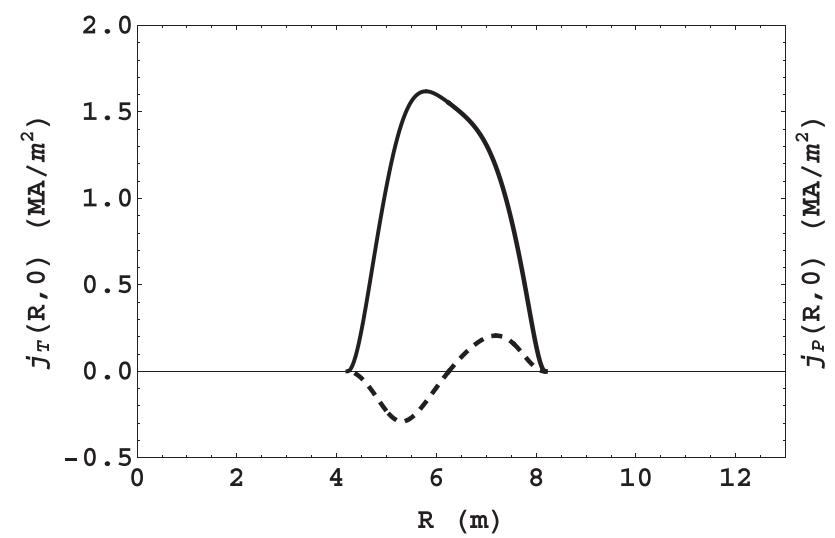

FIG. 7. Profiles of the toroidal (continuous line) and poloidal (dashed line) plasma current densities along the equatorial plane of the ITER-like equilibrium shown in Fig. 5. The toroidal and poloidal plasma currents are $I_{T}(a)=15 \mathrm{MA}$ and $I_{P}(a)=10.4 \mathrm{MA}$, respectively. The total poloidal current is $I(0)=174.7$ MA on the magnetic axis and $I(a)=164.3 \mathrm{MA}$ at the plasma boundary.
Taking moments of these equations, the self and external flux distributions on the plasma boundary can be written in the form

$$
\begin{aligned}
& \Phi_{\mathrm{self}}(\theta)=\left[L_{\mathrm{self}, 0}^{(s)}+\sum_{n=1}^{6}\left(2 L_{\mathrm{self}, n}^{(s)} \cos n \theta+2 L_{\mathrm{self}, n}^{(a)} \sin n \theta\right)\right] I_{T}(a), \\
& \Phi_{\mathrm{ext}}(\theta)=\Phi_{\mathrm{ext}, 0}^{(s)}+\sum_{n=1}^{6}\left(2 \Phi_{\mathrm{ex}, n}^{(s)} \cos n \theta+2 \Phi_{\mathrm{ext}, n}^{(a)} \sin n \theta\right),
\end{aligned}
$$

where

$$
\begin{gathered}
\Phi_{\mathrm{ext}, n}^{(s)}=\sum_{s=1}^{6} L_{s, n}^{(s)} I_{s}+\sum_{k=1}^{6} L_{k, n}^{(s)} I_{k}, \\
\Phi_{\mathrm{ext}, n}^{(a)}=\sum_{s=1}^{6} L_{s, n}^{(a)} I_{s}+\sum_{k=1}^{6} L_{k, n}^{(a)} I_{k} .
\end{gathered}
$$

The tokamak plasma symmetric and antisymmetric selfinductance coefficients are defined, respectively, by 


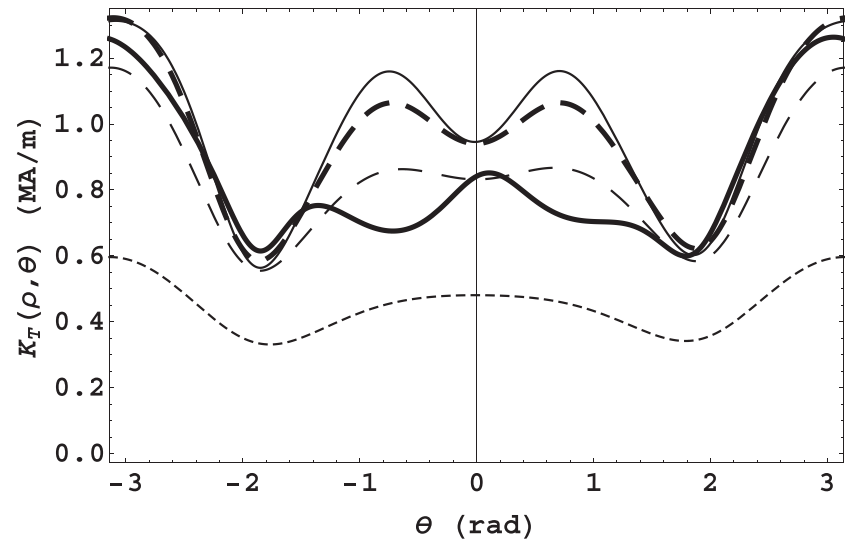

FIG. 8. Equivalent surface current density distribution in the ITER-like tokamak equilibrium shown in Fig. 5. The thick continuous line corresponds to the surface current on the plasma boundary $\rho=a$. The thin lines correspond to the surface current on the flux surfaces $\rho=3 a / 4$ (continuous), $\rho=a / 2$ (dashed) and $\rho=a / 4$ (dotted). The thick dashed line corresponds to the matching radius position $\rho_{*}=0.642 a$.

$$
\begin{aligned}
L_{\text {self }, n}^{(s)} & =\frac{1}{I_{T}(a)}\left\langle\Phi_{\text {self }}(\theta) \cos n \theta\right\rangle_{\theta} \\
& =\frac{2 \pi \mu_{0}}{I_{T}(a)}\left\langle\left\langle h_{\theta}\left(\theta^{\prime}\right) K_{T}\left(\theta^{\prime}\right) G\left(\theta ; \theta^{\prime}\right)\right\rangle_{\theta^{\prime}} \cos n \theta\right\rangle_{\theta}, \\
L_{\text {self }, n}^{(a)} & =\frac{1}{I_{T}(a)}\left\langle\Phi_{\text {self }}(\theta) \sin n \theta\right\rangle_{\theta} \\
& =\frac{2 \pi \mu_{0}}{I_{T}(a)}\left\langle\left\langle h_{\theta}\left(\theta^{\prime}\right) K_{T}\left(\theta^{\prime}\right) G\left(\theta ; \theta^{\prime}\right)\right\rangle_{\theta^{\prime}} \sin n \theta\right\rangle_{\theta} .
\end{aligned}
$$

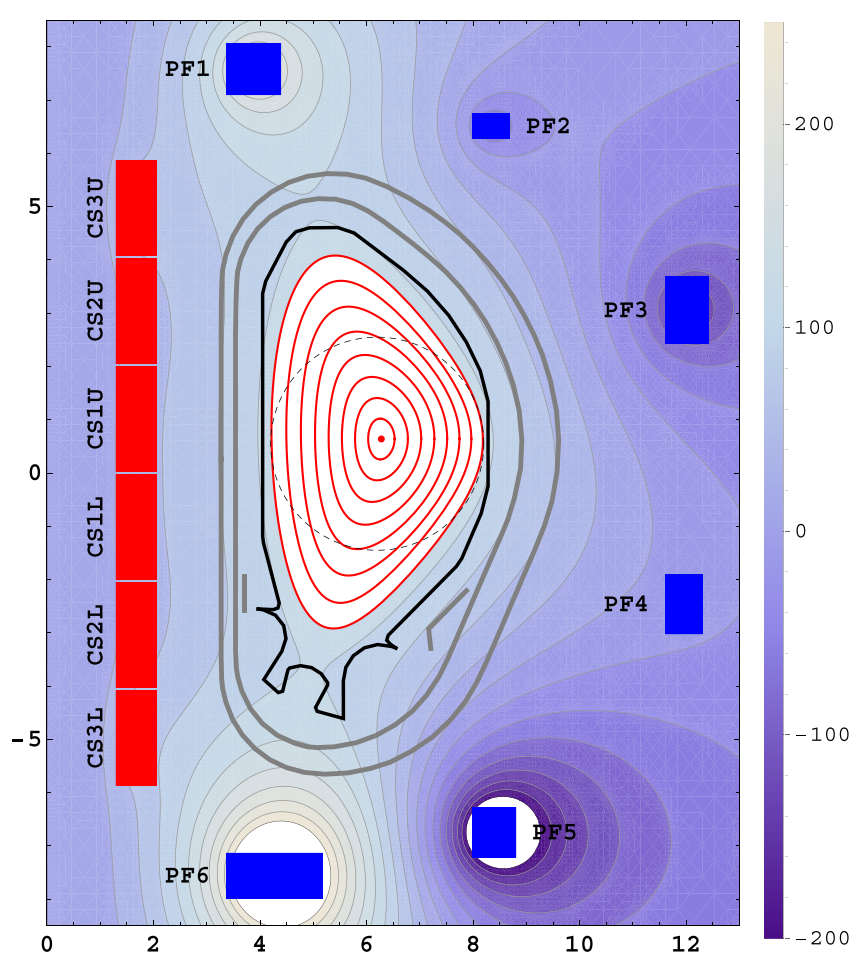

FIG. 9. Contour plot of the vacuum equilibrium magnetic field and plasma flux surfaces for the ITER-like tokamak equilibrium with the adjusted Cauchy condition. The bar legend gives the intensity of the vacuum poloidal flux in $\mathrm{Wb}$, which is displayed with a $25 \mathrm{~Wb}$ interval in the figure.
Accordingly, the mutual inductance coefficients between the tokamak plasma and the central solenoid are defined by

$$
\begin{aligned}
& L_{s, n}^{(s)}=\mu_{0}\left\langle G\left(\theta ; \vec{r}_{s}\right) \cos n \theta\right\rangle_{\theta}, \\
& L_{s, n}^{(a)}=\mu_{0}\left\langle G\left(\theta ; \vec{r}_{s}\right) \sin n \theta\right\rangle_{\theta},
\end{aligned}
$$

and the mutual inductance coefficients between the tokamak plasma and the poloidal field coils by

$$
\begin{aligned}
& L_{k, n}^{(s)}=\mu_{0}\left\langle G\left(\theta ; \vec{r}_{k}\right) \cos n \theta\right\rangle_{\theta}, \\
& L_{k, n}^{(a)}=\mu_{0}\left\langle G\left(\theta ; \vec{r}_{k}\right) \sin n \theta\right\rangle_{\theta} .
\end{aligned}
$$

Formulas to calculate the self-inductance coefficients $L_{\text {self }, n}^{(s)}$ and $L_{\text {self }, n}^{(a)}$ are derived in Appendix A, taking into account the singularity of the Green's function on the plasma boundary.

The Dirichlet condition leads to the following moment equations linking the plasma current to the poloidal field coil currents:

$$
\begin{cases}L_{\text {self }, 0}^{(s)} I_{T}(a)+\underbrace{\sum_{s=1}^{6} L_{s, 0}^{(s)} I_{s}+\sum_{k=1}^{6} L_{k, 0}^{(s)} I_{k}}_{\Phi_{\text {ext, }, 0}^{(s)}}=\Phi(a) & \text { for } n=0 \\ L_{\text {self }, n}^{(s)} I_{T}(a)+\underbrace{\sum_{s=1}^{6} L_{s, n}^{(s)} I_{s}+\sum_{k=1}^{6} L_{k, n}^{(s)} I_{k}}_{\Phi_{\text {ext, }}^{(s)}}=0 & \text { for } n \geqslant 1 \\ L_{\text {self }, n}^{(a)} I_{T}(a)+\underbrace{\sum_{s=1}^{6} L_{s, n}^{(a)} I_{s}+\sum_{k=1}^{6} L_{k, n}^{(a)} I_{k}}_{\Phi_{\text {ex }, n}^{(a)}}=0 & \text { for } n \geqslant 1 .\end{cases}
$$

In ITER, there are twelve independent coils currents: the central solenoid currents $I_{s}$ for $s=1,2, \ldots, 6$, and the poloidal field coils $I_{k}$ for $k=1,2, \ldots, 6$. There is a total of $2 n+1$ equations relating the symmetric and antisymmetric moments (the arbitrary total poloidal flux $\Phi(a)$ at the boundary relates the order zero moments; in the start of burn equilibrium, the total poloidal flux was set equal to zero, $\Phi(a)=0)$. Therefore, it is possible to exactly adjust, in principle, $2 n+1=13$ parameters which describe the plasma boundary. In the present model there are eight Dirichlet parameters $a, R_{0}(a), Z_{0}(a), e(a), S_{2}(a), A_{2}(a), S_{3}(a), A_{3}(a)$ and seven Neumann parameters $R_{0}^{\prime}(a), Z_{0}^{\prime}(a), e^{\prime}(a), S_{2}^{\prime}(a)$, $A_{2}^{\prime}(a), S_{3}^{\prime}(a), A_{3}^{\prime}(a)$, which describe the equivalent surface current density $K_{T}(\theta)$ at the plasma boundary. The major radius $R_{0}(a)$ sets the geometrical scale of the problem, and the total plasma current $I_{T}(a)$ scales the coil currents. One could fix two parameters by anchoring, for example, the outer edge of the plasma cross-section (outer limiter strike point), or the extreme lower point of the plasma (near the " $X$ " point), thereby reducing the number of free parameters. Alternatively, one may discard some of the higher order geometrical corrections. In general, an approximate solution to the system of equations can be determined searching for a minimum of the norm (assuming $2 n+1=13$ symmetric and antisymmetric moments to be determined): 


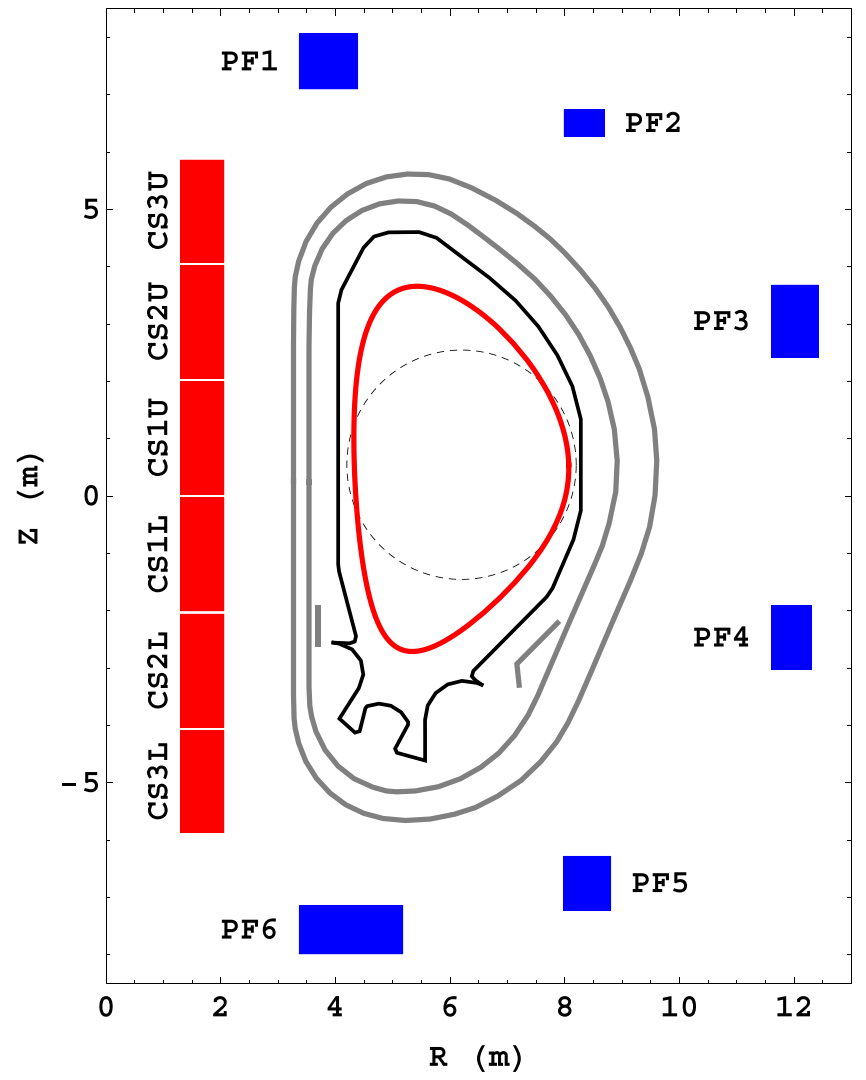

FIG. 10. 95\% flux contour line corresponding to the plasma equilibrium in the start of burn conditions of the ITER tokamak.

$$
\begin{aligned}
\text { Norm }= & \left\{\left(\frac{L_{\text {self }, 0}^{(s)}}{\mu_{0} R_{0}(a)}+\frac{\Phi_{\mathrm{ext}, 0}^{(s)}-\Phi(a)}{\mu_{0} R_{0}(a) I_{T}(a)}\right)^{2}\right. \\
& +\sum_{n=1}^{6}\left[\left(\frac{L_{\mathrm{self}, n}^{(s)}}{\mu_{0} R_{0}(a)}+\frac{\Phi_{\mathrm{ext}, n}^{(s)}}{\mu_{0} R_{0}(a) I_{T}(a)}\right)^{2}\right. \\
& \left.\left.+\left(\frac{L_{\mathrm{self}, n}^{(a)}}{\mu_{0} R_{0}(a)}+\frac{\Phi_{\mathrm{ext}, n}^{(a)}}{\mu_{0} R_{0}(a) I_{T}(a)}\right)^{2}\right]\right\}^{1 / 2} .
\end{aligned}
$$

The minimization of the norm is the indicated method to solve this highly nonlinear system of equation for the boundary parameters. This is particularly true because there are, in general, more free parameters than independent coil currents. Moreover, the quality of the solution is limited by the numerical precision of the inductance coefficient calculations. In control applications, it is possible to linearize the system of equations by expanding the inductance coefficients around a reference geometrical set of parameters. In this case, one cannot adjust more parameters than the number of independent currents.

Now, it is possible to adjust the free plasma boundary (the surface current density $K_{T}(\theta)$ for that matter) to the external equilibrium field. According to Ref. 13, the coil's currents for the ITER burn equilibrium configuration were determined using a numerical solution of the GradShafranov equation, for a given form of the internal toroidal current distribution (actually, the Ampère equation is solved), and by matching the self and external poloidal fluxes along selected points at the plasma boundary. This method is similar to the procedure carried out in Sec. II of the present article. The plasma boundary shape used in the fixed boundary numerical calculation lies along a separatrix, with a "X" point at the lower position. The separatrix leads to a bifurcation with discontinuities in the containing magnetic field and in the equivalent surface current density. This tangential magnetic field discontinuity at the " $\mathrm{X}$ " point complicates the use of the Neumann condition. In this case, the Dirichlet condition can be used with advantage, since the poloidal flux function remains continuous. In general, the separatrix can be represented by a Fourier descriptor and approximated with a small number of parameters using a low-pass filtering of the shape. Ultimately, this reduces the description to the presently used spectral representation (35), with a higher order, if needed. The initial external equilibrium adjustment can be performed using the exact separatrix, but in the free boundary equilibrium calculations, the shape must be smoothed out unless a very large number of parameters are used (essentially an infinite number). As pointed out in Paper I article, higher order corrections in the plasma shape require very high precision in the calculations and are practically impossible to detect experimentally. In any case, the separatrix is strictly beyond the ideal MHD theory, requiring a resistive layer to be correctly described.

In the case at hand, the shape of the separatrix used in the numerical solution is not known. To simplify the comparison with the previous equilibrium solution, one merely reduces the total plasma current by $5 \%$, which corresponds to a reduction of the same order in the area of the plasma cross-section (the exact equivalence between the total current and the cross-sectional area depends on the current profile). Then, the boundary of the plasma conforms to the ITER burn equilibrium currents, starting with the equilibrium solution listed in Table III. The new equilibrium solution corresponds approximately to the $95 \%$ magnetic flux surface, which is described by the Fourier coefficients listed in Table IV and displayed in Fig. 10. Figure 11 shows the corresponding poloidal flux and equivalent surface current

\begin{tabular}{|c|c|c|c|}
\hline \multicolumn{2}{|c|}{ Dirichlet parameters } & \multicolumn{2}{|c|}{ Neumann parameters } \\
\hline $\begin{array}{l}R_{0}(a)=6.2 \mathrm{~m} \\
a=1.870 \mathrm{~m}\end{array}$ & $\begin{array}{c}Z_{0}(a)=0.553 \mathrm{~m} \\
e(a)=1.659\end{array}$ & $\begin{array}{c}R_{0}^{\prime}(a)=-0.294 \\
e^{\prime}(a)=0.739 \mathrm{~m}^{-1}\end{array}$ & $Z_{0}^{\prime}(a)=0.0115$ \\
\hline$S_{2}(a)=0.116$ & $A_{2}(a)=0.00730$ & $S_{2}^{\prime}(a)=0.103 \mathrm{~m}^{-1}$ & $A_{2}^{\prime}(a)=0.0327 \mathrm{~m}^{-1}$ \\
\hline$S_{3}(a)=-0.00388$ & $A_{3}(a)=-0.0125$ & $S_{3}^{\prime}(a)=-0.0102 \mathrm{~m}^{-1}$ & $A_{3}^{\prime}(a)=-0.00626 \mathrm{~m}^{-1}$ \\
\hline
\end{tabular}

TABLE IV. Dirichlet and Neumann parameters corresponding to the $95 \%$ magnetic flux surface adjusted to the ITER start of a burn reference design with the total plasma current reduced to $I_{T}(a)=14.25 \mathrm{MA}$. 

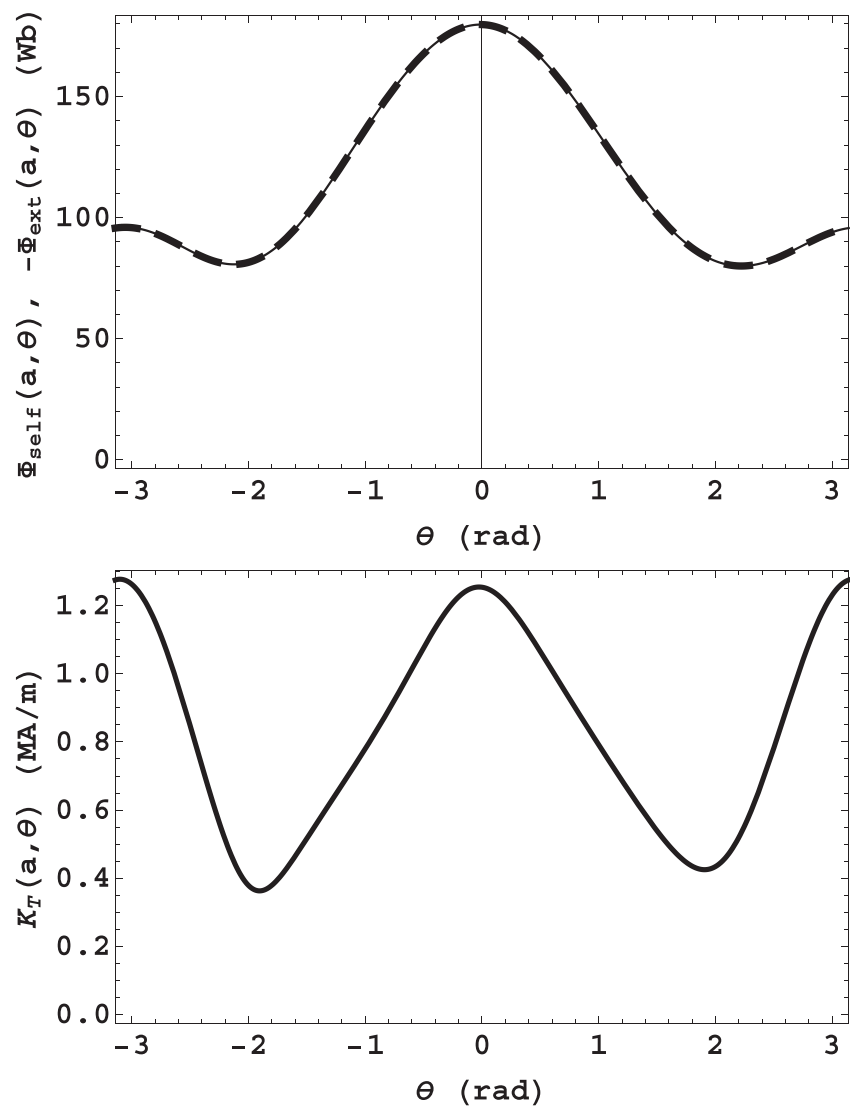

FIG. 11. Poloidal flux distribution (upper graph) and equivalent surface current density distribution (lower graph) at the plasma boundary in the ITERlike tokamak equilibrium shown in Fig. 10. The thick dashed line in the upper graph corresponds to the plasma self-flux distribution, and the thin continuous line corresponds to the flux, taken with the opposite sign, produced on the plasma boundary by the poloidal field coils.

density distributions on this flux surface, which is closely bounded by the separatrix. Although the distribution of currents in the equilibrium field coils in the two cases considered was determined using completely different criteria, the comparison between the free-boundary solutions carried out in the present paper and in Ref. 13 indicates that essentially the same plasma equilibrium is obtained.

\section{CONCLUSIONS}

The first topic covered in this article was the determination of the currents in the poloidal field coils necessary to contain a plasma of given cross-sectional shape. This problem was dealt with by means of well-established procedures based on the virtual-casing principle. The only peculiarity of the present work is the application of suitable spectral representations for the equivalent surface current density on the plasma boundary, obtained using approximate solutions of the internal equilibrium problem presented in Paper I. For a sufficiently large number of external coils, the boundary conditions can be fitted with reasonable precision, and the problem is solved. However, if the fitting is not satisfactory, the equivalent surface current density must be adjusted to satisfy the Cauchy condition imposed on the boundary by the external coils. The initial equilibrium solution is used only to determine the coil currents that approximately contain the desired plasma shape. In the free-boundary application, the spectral representation presents an advantage by providing a number of adjustable parameters that control the plasma shape and the normal gradient to the boundary.

Finally, the adjusted surface current density can be used to determine an approximate, self-consistent analytic solution of the internal equilibrium. This solution is obtained in terms of piecewise continuous approximations of Fourier coefficients in the spectral representation, and a combination of variational moments and direct variational methods. The variational moment method determines the solution in the plasma core, around the magnetic axis, and the direct variational method gives a stationary value of the internal energy consistent with the Cauchy condition imposed by the external coils. In order to satisfy this condition, the direct method bends and stretches the field lines close to the boundary. In this way, the internal current density distribution is determined in consistency with both the imposed and assumed constraints. A comparison between the results of Papers I and II, in particular, for the equivalent current density shown in Figs. 9 and 8 of Papers I and II, respectively, indicates that in order to accommodate the fluctuations in the magnetic field produced by the external coils, the flux surfaces and the current density in the plasma border change, as expected, in a strongly marked way, compared with the changes in the core region. These changes concentrate nearer to the boundary if the matching radius $\rho_{*}$ tends towards the minor plasma radius $a$. This tendency is affected, for example, by the values of the constraints $p^{\prime \prime}(a)$ and $I_{T}^{\prime \prime}(a)$ on the pressure and toroidal current profiles, arbitrarily put equal to zero in Paper I. Obviously, a variety of models can be used to represent possible internal solutions. The true internal equilibrium can be constructed only with additional information provided by transport models and experimental measurements. Nevertheless, the normalized surface current distribution on the plasma boundary is completely defined by the magnetic field produced by the external equilibrium coils.

A simple free-boundary equilibrium solution was determined in Sec. V, using the distribution of currents in the poloidal field coils established for the start of burn conditions in ITER. This calculation was carried out using the Dirichlet moments, in place of the Neumann moments used in Sec. III. Taking into account all the uncertainties, the solution is similar to the fully numerical calculations presented in Ref. 13. With some modifications, the present model can be adapted to represent the plasma closely bounded by the separatrix. The spectral representations are suitable for this application.

The plasma boundary adjustments presented in Secs. III and IV form the main topic of the free-boundary problem. There, it was shown that for a fixed geometry of the external coils and given currents in the coils, the plasma boundary can be determined, and the internal equilibrium as well, taking the total plasma current and major radius as input. Similar equilibrium solutions are generated by scaling these two quantities (this is a consequence of the linearity of Maxwell's equations). This method with proper modifications should be useful for simulating the plasma evolution, in magnetic reconstruction procedures, and in the development of control techniques. In these applications, the Fourier coefficients in the spectral representation act like virtual filaments. 


\section{ACKNOWLEDGMENTS}

This work was supported by a grant provided by the Programa Pesquisador Visitante Sênior: Coordenação de Aperfeiçoamento de Pessoal de Nível Superior (CAPES).

\section{APPENDIX A: SELF-INDUCTANCE COEFFICIENTS}

The Green's function for the axisymmetric Ampères' equation attains its most compact form in cylindrical coordinates $(R, \varphi, Z)$

$$
G\left(\vec{r} ; \vec{r}^{\prime}\right)=\left\langle\frac{\pi R R^{\prime} \cos \left(\varphi-\varphi^{\prime}\right)}{\left|\vec{r}-\vec{r}^{\prime}\right|}\right\rangle_{\varphi^{\prime}}=\sqrt{R R^{\prime}} Q_{1 / 2}(\chi),
$$

where $Q_{1 / 2}$ is a toroidal harmonic (odd-half-integer degree Legendre function of the second kind) and

$$
\chi=\frac{R^{2}+R^{\prime 2}+\left(Z^{2}-Z^{\prime 2}\right)}{2 R R^{\prime}} \quad(1 \leqslant \chi<\infty) .
$$

Putting $\chi=(2-m) / m$, and using the relation

$$
Q_{1 / 2}\left(\frac{2-m}{m}\right)=\frac{(2-m) K(m)-2 E(m)}{\sqrt{m}}
$$

the usual expression in terms of complete elliptic integrals $K(m)$ and $E(m)$ is recovered

$$
G\left(R, Z ; R^{\prime}, Z^{\prime}\right)=\sqrt{R R^{\prime}}\left(\frac{(2-m) K(m)-2 E(m)}{\sqrt{m}}\right),
$$

with

$$
m=\frac{4 R R^{\prime}}{\left(R+R^{\prime}\right)^{2}+\left(Z-Z^{\prime}\right)^{2}} \quad(0 \leqslant m<1)
$$

The plasma boundary is defined parametrically in the form

$$
\begin{cases}R=R(\theta), & Z=Z(\theta) \\ R^{\prime}=R\left(\theta^{\prime}\right), & Z^{\prime}=Z\left(\theta^{\prime}\right) .\end{cases}
$$

Taking into account the singular character of the Green's function

$$
G\left(\theta ; \theta^{\prime}\right) \underset{\theta^{\prime} \rightarrow \theta}{\rightarrow}-h_{\zeta}(a, \theta)\left\{\frac{1}{2} \ln \left[\left(\frac{h_{\theta}(a, \theta)}{8 h_{\zeta}(a, \theta)}\right) 2 \sin \left(\frac{\theta-\theta^{\prime}}{2}\right)\right]^{2}+2\right\},
$$

the nonsingular auxiliary function $g\left(\theta ; \theta^{\prime}\right)$ is defined as

$$
\begin{aligned}
g\left(\theta ; \theta^{\prime}\right)= & \frac{G\left(\theta ; \theta^{\prime}\right)}{h_{\zeta}(a, \theta)}+\left\{\frac { 1 } { 2 } \operatorname { l n } \left[\left(\frac{h_{\theta}(a, \theta)}{8 h_{\zeta}(a, \theta)}\right)\right.\right. \\
& \left.\left.\times 2 \sin \left(\frac{\theta-\theta^{\prime}}{2}\right)\right]^{2}+2\right\} \underset{\theta^{\prime} \rightarrow \theta}{\rightarrow} 0 .
\end{aligned}
$$

Using the integral

$$
\left\langle\frac{1}{2} \ln \left[2 \sin \left(\frac{\theta-\theta^{\prime}}{2}\right)\right]^{2}\right\rangle_{\theta^{\prime}}=0 \quad(0 \leqslant \theta \leqslant 2 \pi)
$$

the expression for the external self-inductance of the tokamak plasma becomes ${ }^{14}$

$$
\begin{aligned}
L_{e}(a)= & \frac{1}{K(a)}\left\langle\left(\frac{h_{\theta}^{2}(a, \theta) h_{\zeta}(a, \theta)}{\sqrt{g}(a, \theta)}\right)\left\langle g\left(\theta ; \theta^{\prime}\right)\right\rangle_{\theta^{\prime}}\right\rangle_{\theta} \\
& +\frac{1}{K(a)}\left\langle\left(\frac{h_{\theta}^{2}(a, \theta) h_{\zeta}(a, \theta)}{\sqrt{g}(a, \theta)}\right)\left[\ln \left(\frac{8 h_{\zeta}(a, \theta)}{h_{\theta}(a, \theta)}\right)-2\right]\right\rangle_{\theta},
\end{aligned}
$$

with the singularity analytically removed.

The singularity in the Green's function can also be handled by evaluating the self-field contribution in the form

$$
\begin{aligned}
G_{0}(\theta)= & \frac{1}{2 \pi} \int_{0}^{\theta-\epsilon} G\left(\theta, \theta^{\prime}\right) d \theta^{\prime}+\frac{1}{2 \pi} \int_{\theta+\epsilon}^{2 \pi} G\left(\theta, \theta^{\prime}\right) d \theta^{\prime} \\
& +\frac{\epsilon}{\pi} h_{\zeta}(a, \theta)\left[\ln \left(\frac{8 h_{\zeta}(a, \theta)}{\epsilon h_{\theta}(a, \theta)}\right)-1\right] \\
& \underset{\epsilon \rightarrow 0}{\rightarrow} \frac{P V}{2 \pi} \int_{0}^{2 \pi} G\left(\theta, \theta^{\prime}\right) d \theta^{\prime},
\end{aligned}
$$

so that the external inductance is calculated, for a sufficiently small value of $\epsilon$, by

$$
L_{e}(a)=\frac{1}{K(a)}\left\langle\left(\frac{h_{\theta}^{2}(a, \theta)}{\sqrt{g}(a, \theta)}\right)_{a} G_{0}(\theta)\right\rangle_{\theta} .
$$

The symmetric and antisymmetric moments of the nonsingular auxiliary function $g\left(\theta ; \theta^{\prime}\right)$ are defined, respectively, by

$$
\begin{aligned}
& g_{n}^{(s)}(\theta)=\left\langle g\left(\theta ; \theta^{\prime}\right) \cos n \theta^{\prime}\right\rangle_{\theta^{\prime}}, \\
& g_{n}^{(a)}(\theta)=\left\langle g\left(\theta ; \theta^{\prime}\right) \sin n \theta^{\prime}\right\rangle_{\theta^{\prime}} .
\end{aligned}
$$

The expressions for the tokamak plasma self-inductance coefficients defined in Sec. V become

$$
\begin{aligned}
L_{\mathrm{self}, n}^{(s)}= & \frac{1}{K(a)}\left\langle\left\langle\frac{h_{\theta}^{2}(a, \theta)}{\sqrt{g}(a, \theta)} G\left(\theta ; \theta^{\prime}\right) \cos n \theta^{\prime}\right\rangle_{\theta^{\prime}}\right\rangle_{\theta} \\
= & \frac{1}{K(a)}\left\langle\frac{h_{\theta}^{2}(a, \theta) h_{\zeta}(a, \theta)}{\sqrt{g}(a, \theta)} g_{n}^{(s)}(\theta)\right\rangle_{\theta} \\
& -\frac{1}{K(a)}\left\langle\frac{h_{\theta}^{2}(a, \theta) h_{\zeta}(a, \theta)}{\sqrt{g}(a, \theta)}\right. \\
& \times\left\langle\left\{\frac{1}{2} \ln \left[\left(\frac{h_{\theta}(a, \theta)}{8 h_{\zeta}(a, \theta)}\right) 2 \sin \left(\frac{\theta-\theta^{\prime}}{2}\right)\right]^{2}+2\right\}\right. \\
& \left.\left.\times \cos n \theta^{\prime}\right\rangle_{\theta^{\prime}}\right\rangle_{\theta},
\end{aligned}
$$

and 


$$
\begin{aligned}
L_{\text {self }, n}^{(a)}= & \frac{1}{K(a)}\left\langle\left\langle\frac{h_{\theta}^{2}(a, \theta)}{\sqrt{g}(a, \theta)} G\left(\theta ; \theta^{\prime}\right) \sin n \theta^{\prime}\right\rangle_{\theta^{\prime}}\right\rangle_{\theta} \\
= & \frac{1}{K(a)}\left\langle\frac{h_{\theta}^{2}(a, \theta) h_{\zeta}(a, \theta)}{\sqrt{g}(a, \theta)} g_{n}^{(s)}(\theta)\right\rangle_{\theta} \\
& -\frac{1}{K(a)}\left\langle\frac{h_{\theta}^{2}(a, \theta) h_{\zeta}(a, \theta)}{\sqrt{g}(a, \theta)}\right. \\
& \times\left\langle\left\{\frac{1}{2} \ln \left[\left(\frac{h_{\theta}(a, \theta)}{8 h_{\zeta}(a, \theta)}\right) 2 \sin \left(\frac{\theta-\theta^{\prime}}{2}\right)\right]^{2}+2\right\}\right. \\
& \left.\left.\times \sin n \theta^{\prime}\right\rangle_{\theta^{\prime}}\right\rangle_{\theta} .
\end{aligned}
$$

Using the integrals

$$
\begin{aligned}
& \frac{1}{4 \pi} \int_{0}^{2 \pi} \ln \left[2 \sin \left(\frac{\theta-\theta^{\prime}}{2}\right)\right]^{2} \cos n \theta^{\prime} d \theta^{\prime}=-\frac{\cos n \theta}{2 n}\left(1-\delta_{n, 0}\right), \\
& \frac{1}{4 \pi} \int_{0}^{2 \pi} \ln \left[2 \sin \left(\frac{\theta-\theta^{\prime}}{2}\right)\right]^{2} \sin n \theta^{\prime} d \theta^{\prime}=-\frac{\sin n \theta}{2 n}\left(1-\delta_{n, 0}\right),
\end{aligned}
$$

it follows that

$$
\begin{aligned}
L_{\text {self }, n}^{(s)}= & \frac{1}{K(a)}\left\langle\frac { h _ { \theta } ^ { 2 } ( a , \theta ) h _ { \zeta } ( a , \theta ) } { \sqrt { g } ( a , \theta ) } \left[ g_{0}^{(s)}(\theta)+\ln \left(\frac{8 h_{\zeta}(a, \theta)}{h_{\theta}(a, \theta)}\right)\right.\right. \\
& -2]\rangle_{\theta} \delta_{n, 0}+\frac{1}{K(a)}\left\langle\frac{h_{\theta}^{2}(a, \theta) h_{\zeta}(a, \theta)}{\sqrt{g}(a, \theta)}\right. \\
& \left.\times\left[g_{n}^{(s)}(\theta)+\frac{\cos n \theta}{2 n}\right]\right\rangle_{\theta}\left(1-\delta_{n, 0}\right),
\end{aligned}
$$

and

$L_{\mathrm{self}, n}^{(a)}=\frac{1}{K(a)}\left\langle\frac{h_{\theta}^{2}(a, \theta) h_{\zeta}(a, \theta)}{\sqrt{g}(a, \theta)}\left[g_{n}^{(a)}(\theta)+\frac{\sin n \theta}{2 n}\right]\right\rangle_{\theta}\left(1-\delta_{n, 0}\right)$.

The symmetric self-inductance coefficient of order zero gives the external self-inductance of the plasma, i.e., $L_{\mathrm{self}, 0}^{(s)}=L_{e}(a)$.

\section{APPENDIX B: NEUMANN COEFFICIENTS}

The normal unit vector to a two-dimensional curve given in a parametric form $R=R(\theta), Z=Z(\theta)$ is

$$
\hat{n}(\theta)=\operatorname{sign}\left[\kappa_{c}(\theta)\right]\left(\frac{-(\partial Z / \partial \theta) \nabla R+(\partial R / \partial \theta) \nabla Z}{\sqrt{(\partial R / \partial \theta)^{2}+(\partial Z / \partial \theta)^{2}}}\right),
$$

where $\kappa_{c}(\theta)$ is the curvature

$$
\kappa_{c}(\theta)=\frac{(\partial R / \partial \theta)\left(\partial^{2} Z / \partial \theta^{2}\right)-(\partial Z / \partial \theta)\left(\partial^{2} R / \partial \theta^{2}\right)}{\left[(\partial R / \partial \theta)^{2}+(\partial Z / \partial \theta)^{2}\right]^{3 / 2}} .
$$

Therefore, the normal component of $\nabla G$ can be put in the form

$$
\begin{aligned}
\hat{n} \cdot \nabla G\left(\theta ; R^{\prime}, Z^{\prime}\right)= & \frac{\operatorname{sign}\left[\kappa_{c}(\theta)\right]}{\sqrt{(\partial R / \partial \theta)^{2}+(\partial Z / \partial \theta)^{2}}} \\
& \times\left(-\frac{\partial Z}{\partial \theta} \frac{\partial G}{\partial R}+\frac{\partial R}{\partial \theta} \frac{\partial G}{\partial Z}\right),
\end{aligned}
$$

and the external set of poloidal coils creates a magnetic field component, tangential to the plasma boundary $\ell_{P}(a)$, given by

$$
\begin{aligned}
B_{\tau, \text { ext }}(\theta)= & -\frac{\mu_{0}}{2 \pi h_{\zeta}(\theta)} \sum_{k} I_{k} \hat{n} \cdot \nabla G\left(\theta ; \vec{r}_{k}\right) \\
= & -\frac{\mu_{0} \operatorname{sign}\left[\kappa_{c}(\theta)\right]}{2 \pi R(\theta) \sqrt{(\partial R / \partial \theta)^{2}+(\partial Z / \partial \theta)^{2}}} \\
& \times \sum_{k} I_{k}\left(-\frac{\partial Z}{\partial \theta} \frac{\partial G\left(\theta ; \vec{r}_{k}\right)}{\partial R}+\frac{\partial R}{\partial \theta} \frac{\partial G\left(\theta ; \vec{r}_{k}\right)}{\partial Z}\right) .
\end{aligned}
$$

In this expression, the partial derivatives of $G\left(R, Z ; R^{\prime}, Z^{\prime}\right)$ with respect to $R$ and $Z$ are calculated with the help of the formulas

$$
\left\{\begin{array}{l}
\frac{\partial G\left(R, Z ; R^{\prime}, Z^{\prime}\right)}{\partial R}=\frac{\sqrt{m}}{2}\left\{K(m)-\left[1-\left(1+\frac{R^{\prime}}{R}\right) \frac{m}{2}\right] \frac{E(m)}{1-m}\right\} \sqrt{\frac{R}{R^{\prime}}} \\
\frac{\partial G\left(R, Z ; R^{\prime}, Z^{\prime}\right)}{\partial Z}=\frac{\sqrt{m}}{2}\left[K(m)-\left(1-\frac{m}{2}\right) \frac{E(m)}{1-m}\right] \frac{Z-Z^{\prime}}{\sqrt{R R^{\prime}}} .
\end{array}\right.
$$

Using the limiting values

$$
\begin{aligned}
& \frac{\partial G\left(\theta ; \theta^{\prime}\right)}{\partial R \quad \underset{\theta^{\prime} \rightarrow \theta}{\rightarrow}}-\frac{R(\theta) \partial R / \partial \theta}{\left[(\partial R / \partial \theta)^{2}+(\partial Z / \partial \theta)^{2}\right]\left(\theta-\theta^{\prime}\right)} \\
&+\frac{1}{4} \ln \left[\frac{64 R^{2}(\theta)}{\left[(\partial R / \partial \theta)^{2}+(\partial Z / \partial \theta)^{2}\right]\left(\theta-\theta^{\prime}\right)^{2}}\right] \\
&+ \frac{1 / 2}{\left[(\partial R / \partial \theta)^{2}+(\partial Z / \partial \theta)^{2}\right]^{2}} R(\theta)\left(\frac{\partial Z}{\partial \theta}\right)^{2} \frac{\partial^{2} R}{\partial \theta^{2}} \\
&-\left(\frac{\partial R}{\partial \theta}\right)^{2}\left[\left(\frac{\partial Z}{\partial \theta}\right)^{2}+R(\theta) \frac{\partial^{2} R}{\partial \theta^{2}}\right] \\
&\left.-2 R(\theta) \frac{\partial R}{\partial \theta} \frac{\partial Z}{\partial \theta} \frac{\partial^{2} Z}{\partial \theta^{2}}-\left(\frac{\partial Z}{\partial \theta}\right)^{4}\right\}
\end{aligned}
$$




$$
\begin{aligned}
\frac{\partial G\left(\theta ; \theta^{\prime}\right)}{\partial Z} \underset{\theta^{\prime} \rightarrow \theta}{\rightarrow} & -\frac{R(\theta) \partial Z / \partial \theta}{\left[(\partial R / \partial \theta)^{2}+(\partial Z / \partial \theta)^{2}\right]\left(\theta-\theta^{\prime}\right)} \\
& +\frac{1 / 2}{\left[(\partial R / \partial \theta)^{2}+(\partial Z / \partial \theta)^{2}\right]^{2}}\left\{\frac { \partial R } { \partial \theta } \frac { \partial Z } { \partial \theta } \left[\left(\frac{\partial R}{\partial \theta}\right)^{2}\right.\right. \\
& \left.+\left(\frac{\partial Z}{\partial \theta}\right)^{2}-2 R(\theta) \frac{\partial^{2} R}{\partial \theta^{2}}\right]+R(\theta)\left[\left(\frac{\partial R}{\partial \theta}\right)^{2}\right. \\
& \left.\left.-\left(\frac{\partial Z}{\partial \theta}\right)^{2}\right] \frac{\partial^{2} Z}{\partial \theta^{2}}\right\}
\end{aligned}
$$

the limiting value of the normal component of $\nabla G$ can be written as

$$
\begin{aligned}
\lim _{\theta^{\prime} \rightarrow \theta} \hat{n} \cdot \nabla G\left(\theta ; \theta^{\prime}\right)= & \frac{\sqrt{h_{\theta}^{2}(\theta)-\left(\partial h_{\zeta} / \partial \theta\right)^{2}}}{2 h_{\theta}(\theta)} \\
& \times \operatorname{sign}\left(\frac{\partial h_{\theta}}{\partial \theta} \frac{\partial h_{\zeta}}{\partial \theta}-h_{\theta}(\theta) \frac{\partial^{2} h_{\zeta}}{\partial \theta^{2}}\right) \\
& \times\left\{\frac{1}{2} \ln \left[\left(\frac{h_{\theta}(\theta)}{8 h_{\zeta}(\theta)}\right) 2 \sin \left(\frac{\theta-\theta^{\prime}}{2}\right)\right]^{2}+1\right. \\
& +\frac{h_{\zeta}(\theta) / h_{\theta}(\theta)}{h_{\theta}^{2}(\theta)-\left(\partial h_{\zeta} / \partial \theta\right)^{2}}\left(\frac{\partial h_{\theta}}{\partial \theta} \frac{\partial h_{\zeta}}{\partial \theta}\right. \\
& \left.\left.-h_{\theta}(\theta) \frac{\partial^{2} h_{\zeta}}{\partial \theta^{2}}\right)\right\}
\end{aligned}
$$

The sign $\left[\left(\partial h_{\theta} / \partial \theta\right) \partial h_{\zeta} / \partial \theta-h_{\theta}(\theta) \partial^{2} h_{\zeta} / \partial \theta^{2}\right]= \pm 1$ variable is related to the extremum points of the closed poloidal contour, along the $Z$ axis. The singularity of the normal component of the Green's function gradient can be handled introducing the above limit in the expression of the containing field calculated from the equivalent surface current density on the plasma boundary. Hence, the containing field can be written in the form

$$
\begin{aligned}
B_{\tau, \text { ext }}(\theta)= & -\frac{\mu_{0} K_{T}(\theta)}{2}-\mu_{0} K_{T}(\theta) \frac{\sqrt{h_{\theta}^{2}(\theta)-\left(\partial h_{\zeta} / \partial \theta\right)^{2}}}{2 h_{\zeta}(\theta)} \\
& \times \operatorname{sign}\left(\frac{\partial h_{\theta}}{\partial \theta} \frac{\partial h_{\zeta}}{\partial \theta}-h_{\theta}(\theta) \frac{\partial^{2} h_{\zeta}}{\partial \theta^{2}}\right)\left[\ln \left(\frac{8 h_{\zeta}(\theta)}{h_{\theta}(\theta)}\right)\right. \\
& \left.-1-\frac{h_{\zeta}(\theta) / h_{\theta}(\theta)}{h_{\theta}^{2}(\theta)-\left(\partial h_{\zeta} / \partial \theta\right)^{2}}\left(\frac{\partial h_{\theta}}{\partial \theta} \frac{\partial h_{\zeta}}{\partial \theta}-h_{\theta}(\theta) \frac{\partial^{2} h_{\zeta}}{\partial \theta^{2}}\right)\right] \\
& +\frac{\mu_{0}}{h_{\zeta}(\theta)}\left\langle h_{\theta}\left(\theta^{\prime}\right) K_{T}\left(\theta^{\prime}\right) \hat{n} \cdot \nabla G\left(\theta ; \theta^{\prime}\right)\right. \\
& \left.-h_{\theta}(\theta) K_{T}(\theta) \lim _{\theta^{\prime} \rightarrow \theta} \hat{n} \cdot \nabla G\left(\theta ; \theta^{\prime}\right)\right\rangle_{\theta^{\prime}}
\end{aligned}
$$

Introducing the dimensionless auxiliary function

$$
\begin{aligned}
n\left(\theta ; \theta^{\prime}\right)= & \left(\frac{h_{\theta}^{2}\left(\theta^{\prime}\right)}{\sqrt{g}\left(\theta^{\prime}\right)}\right) \hat{n} \cdot \nabla G\left(\theta ; \theta^{\prime}\right) \\
& -\left(\frac{h_{\theta}^{2}(\theta)}{\sqrt{g}(\theta)}\right) \lim _{\theta^{\prime} \rightarrow \theta}\left[\hat{n} \cdot \nabla G\left(\theta ; \theta^{\prime}\right)\right] \underset{\theta^{\prime} \rightarrow \theta}{\rightarrow} 0
\end{aligned}
$$

the system of equations relating the currents in the poloidal field coils and the plasma current can be written in the simplified form given by Eq. (42), where $N_{n}^{(s)}$ denotes the symmetric

$$
\begin{aligned}
N_{n}^{(s)}= & \frac{1}{\mu_{0} K(a)}\left\langle( \frac { h _ { \zeta } ( \theta ) h _ { \theta } ( \theta ) } { \sqrt { g } ( \theta ) } ) \left\{\frac{\cos (n \theta)}{2}\right.\right. \\
& +\frac{\sqrt{h_{\theta}^{2}(\theta)-\left(\partial h_{\zeta} / \partial \theta\right)^{2}}}{2 h_{\zeta}(\theta)} \operatorname{sign}\left(\frac{\partial h_{\theta}}{\partial \theta} \frac{\partial h_{\zeta}}{\partial \theta}-h_{\theta}(\theta) \frac{\partial^{2} h_{\zeta}}{\partial \theta^{2}}\right) \\
& \times \cos (n \theta)\left[\ln \left(\frac{8 h_{\zeta}(\theta)}{h_{\theta}(\theta)}\right)-1-\frac{h_{\zeta}(\theta) / h_{\theta}(\theta)}{h_{\theta}^{2}(\theta)-\left(\partial h_{\zeta} / \partial \theta\right)^{2}}\right. \\
& \left.\left.\left.\times\left(\frac{\partial h_{\theta}}{\partial \theta} \frac{\partial h_{\zeta}}{\partial \theta}-h_{\theta}(\theta) \frac{\partial^{2} h_{\zeta}}{\partial \theta^{2}}\right)\right]\right\}\right\rangle_{\theta}-\frac{1}{\mu_{0} K(a)} \\
& \times\left\langle\left\langle n\left(\theta ; \theta^{\prime}\right)\right\rangle_{\theta^{\prime}} \cos (n \theta)\right\rangle_{\theta},
\end{aligned}
$$

and $N_{n}^{(a)}$ the antisymmetric Neumann coefficients of order $n$ for the plasma boundary

$$
\begin{aligned}
N_{n}^{(a)}= & \frac{1}{\mu_{0} K(a)}\left\langle( \frac { h _ { \zeta } ( \theta ) h _ { \theta } ( \theta ) } { \sqrt { g } ( \theta ) } ) \left\{\frac{\sin (n \theta)}{2}\right.\right. \\
& +\frac{\sqrt{h_{\theta}^{2}(\theta)-\left(\partial h_{\zeta} / \partial \theta\right)^{2}}}{2 h_{\zeta}(\theta)} \operatorname{sign}\left(\frac{\partial h_{\theta}}{\partial \theta} \frac{\partial h_{\zeta}}{\partial \theta}-h_{\theta}(\theta) \frac{\partial^{2} h_{\zeta}}{\partial \theta^{2}}\right) \\
& \times \sin (n \theta)\left[\ln \left(\frac{8 h_{\zeta}(\theta)}{h_{\theta}(\theta)}\right)-1-\frac{h_{\zeta}(\theta) / h_{\theta}(\theta)}{h_{\theta}^{2}(\theta)-\left(\partial h_{\zeta} / \partial \theta\right)^{2}}\right. \\
& \left.\left.\left.\times\left(\frac{\partial h_{\theta}}{\partial \theta} \frac{\partial h_{\zeta}}{\partial \theta}-h_{\theta}(\theta) \frac{\partial^{2} h_{\zeta}}{\partial \theta^{2}}\right)\right]\right\}\right\rangle_{\theta} \\
& -\frac{1}{\mu_{0} K(a)}\left\langle\left\langle n\left(\theta ; \theta^{\prime}\right)\right\rangle_{\theta^{\prime}} \sin (n \theta)\right\rangle_{\theta} .
\end{aligned}
$$

The magnetic field produced by some of the poloidal field coils in a tokamak configuration may be approximated using the flux produced by thin cylindrical solenoids. This approximation is used in the present article to calculate the field produced by the segments of the central solenoid in the ITER-like tokamak. In cylindrical coordinates $(R, Z)$, the poloidal flux produced by a thin solenoid of radius $R_{s}$ and total length $\Delta Z_{s}$ centered at the vertical position $Z_{s}$ is given in terms of complete elliptic integrals of the first, second and third kinds, $K, E$ and $\Pi$, respectively,

$$
\begin{aligned}
\Phi_{s}\left(R, Z ; R_{s}, Z_{s}, \Delta Z_{s}\right) \\
=\mu_{0} I_{s} \frac{\sqrt{R R_{s}}}{\Delta Z_{s}}\left\{\frac { Z - Z _ { s } - \Delta Z _ { s } / 2 } { \sqrt { m _ { s } ^ { ( + ) } } } \left[E\left(m_{s}^{(+)}\right)-K\left(m_{s}^{(+)}\right)\right.\right. \\
\left.\quad+m_{s}^{(+)}\left(\frac{1}{n_{s}}-1\right)\left(\Pi\left(n_{s}, m_{s}^{(+)}\right)-K\left(m_{s}^{(+)}\right)\right)\right] \\
\quad-\frac{Z-Z_{s}+\Delta Z_{s} / 2}{\sqrt{m_{s}^{(-)}}}\left[E\left(m_{s}^{(-)}\right)-K\left(m_{s}^{(-)}\right)+m_{s}^{(-)}\left(\frac{1}{n_{s}}-1\right)\right. \\
\left.\left.\quad \times\left(\Pi\left(n_{s, m_{s}^{(-)}}\right)-K\left(m_{s}^{(-)}\right)\right)\right]\right\},
\end{aligned}
$$


where $I_{s}$ is the total current flowing in the solenoid, and

$$
\begin{cases}m_{s}^{( \pm)}=\frac{4 R R_{s}}{\left(R+R_{s}\right)^{2}+\left(Z-\left(Z_{s} \pm \Delta Z_{s} / 2\right)\right)^{2}} & \left(0 \leqslant m_{s}^{( \pm)}<1\right) \\ n_{s}=\frac{4 R R_{s}}{\left(R+R_{s}\right)^{2}} & \left(0 \leqslant n_{s} \leqslant 1\right) .\end{cases}
$$

The partial derivatives of $\Phi_{s}$ with respect to $R$ and $Z$ are

$$
\begin{aligned}
\frac{\partial \Phi_{s}}{\partial R}= & \mu_{0} I_{s} \frac{\sqrt{R R_{s}}}{\Delta Z_{s}}\left\{\frac{Z-Z_{s}+\Delta Z_{s} / 2}{2 R_{s}} \sqrt{m_{s}^{(-)}}\right. \\
& \times\left[K\left(m_{s}^{(-)}\right)-\left(\frac{R-R_{s}}{R+R_{s}}\right) \Pi\left(n_{s}, m_{s}^{(-)}\right)\right] \\
& -\frac{Z-Z_{s}-\Delta Z_{s} / 2}{2 R_{s}} \sqrt{m_{s}^{(+)}}\left[K\left(m_{s}^{(+)}\right)\right. \\
& \left.\left.-\left(\frac{R-R_{s}}{R+R_{s}}\right) \Pi\left(n_{s}, m_{s}^{(+)}\right)\right]\right\}
\end{aligned}
$$

and

$$
\begin{aligned}
\frac{\partial \Phi_{s}}{\partial Z}= & \mu_{0} I_{s} \frac{\sqrt{R R_{s}}}{\Delta Z_{s}}\left\{\frac { 1 } { \sqrt { m _ { s } ^ { ( - ) } } } \left[\left(2-m_{s}^{(-)}\right) K\left(m_{s}^{(-)}\right)\right.\right. \\
& \left.-2 E\left(m_{s}^{(-)}\right)\right]-\frac{1}{\sqrt{m_{s}^{(+)}}}\left[\left(2-m_{s}^{(+)}\right) K\left(m_{s}^{(+)}\right)\right. \\
& \left.\left.-2 E\left(m_{s}^{(+)}\right)\right]\right\} .
\end{aligned}
$$

${ }^{1}$ G. O. Ludwig, "Analytical solution of the tokamak equilibrium. I. The fixed-boundary case," Phys. Plasma 24, 092502 (2017).

${ }^{2}$ V. D. Shafranov and L. E. Zakharov, "Use of the virtual-casing principle in calculating the containing magnetic field in toroidal plasma systems," Nucl. Fusion 12, 599-601 (1972).

${ }^{3}$ L. E. Zhakarov, "Numerical methods for solving some problems of the theory of plasma equilibrium in toroidal configurations," Nucl. Fusion 13, 595-602 (1973).

${ }^{4}$ L. E. Zakharov and V. D. Shafranov, "Equilibrium of current-carrying plasmas in toroidal configurations," Rev. Plasma Phys. 11, 153-308 (1986).

${ }^{5}$ K. Sakurai, Y. Tanaka, and T. Okuda, "Equilibrium in an octopole tokamak without conducting shell," Plasma Phys. 17, 261-269 (1975).

${ }^{6}$ J. P. Goedbloed, "Free-boundary high-beta tokamaks. I. Free-boundary equilibrium," Phys. Fluids 25, 852-868 (1982).

${ }^{7}$ J. P. Goedbloed, R. Keppens, and S. Poedts, Advanced Magnetohydrodynamics (Cambridge University Press, Cambridge, 2010), Part IV.

${ }^{8} \mathrm{~F}$. Alladio and F. Crisanti, "Analysis of MHD equilibria by toroidal multipolar expansions," Nucl. Fusion 26, 1143-1164 (1986).

${ }^{9}$ K. Lackner, "Computation of ideal MHD equilibria," Comput. Phys. Commun. 12, 33-44 (1976).

${ }^{10}$ J. L. Johnson, H. E. Dalhed, J. M. Greene, R. C. Grimm, Y. Y. Hsieh, S. C. Jardin, J. Manickam, M. Okabayashi, R. G. Storer, A. M. M. Todd, D. E. Voss, and K. E. Weimer, "Numerical determination of axisymmetric toroidal magnetohydrodynamic equilibria," J. Comput. Phys. 32, 212-234 (1979).

${ }^{11}$ S. Jardin, Computational Methods in Plasma Physics (CRC Press, Boca Raton, 2010), Chap. 4.

${ }^{12}$ G. O. Ludwig, P. Rodrigues, and J. P. S. Bizarro, "Tokamak equilibria with strong toroidal current density reversal," Nucl. Fusion 53, 053001 (2013).

${ }^{13}$ A. Portone, "Perturbed solutions of fixed boundary MHD equilibria," Nucl. Fusion 44, 265-278 (2004).

${ }^{14}$ G. O. Ludwig and M. C. R. Andrade, "External inductance of large to ultralow aspect-ratio tokamak plasmas," Phys. Plasmas 5, 2274-2283 (1998). 